\title{
Stellar encounters with the solar system
}

\author{
J. García-Sánchez ${ }^{1}$, P. R. Weissman ${ }^{2}$, R. A. Preston ${ }^{2}$, D. L. Jones ${ }^{2}$, J.-F. Lestrade ${ }^{3}$, D. W. Latham ${ }^{4}$ \\ R. P. Stefanik ${ }^{4}$, and J. M. Paredes ${ }^{1}$ \\ 1 Departament d'Astronomia i Meteorologia, Universitat de Barcelona, Av. Diagonal 647, 08028 Barcelona, Spain \\ 2 Jet Propulsion Laboratory, California Institute of Technology, 4800 Oak Grove Drive, Pasadena, CA 91109, \\ USA \\ 3 Observatoire de Paris/DEMIRM-CNRS8540, 77 Av. Denfert Rochereau, 75014 Paris, France \\ 4 Harvard-Smithsonian Center for Astrophysics, 60 Garden Street, Cambridge, MA 02138, USA
}

Received 20 April 2001 / Accepted 17 September 2001

\begin{abstract}
We continue our search, based on Hipparcos data, for stars which have encountered or will encounter the solar system (García-Sánchez et al. 1999). Hipparcos parallax and proper motion data are combined with ground-based radial velocity measurements to obtain the trajectories of stars relative to the solar system. We have integrated all trajectories using three different models of the galactic potential: a local potential model, a global potential model, and a perturbative potential model. The agreement between the models is generally very good. The time period over which our search for close passages is valid is about \pm 10 Myr. Based on the Hipparcos data, we find a frequency of stellar encounters within one parsec of the Sun of $2.3 \pm 0.2$ per Myr. However, we also find that the Hipparcos data is observationally incomplete. By comparing the Hipparcos observations with the stellar luminosity function for star systems within $50 \mathrm{pc}$ of the Sun, we estimate that only about one-fifth of the stars or star systems were detected by Hipparcos. Correcting for this incompleteness, we obtain a value of $11.7 \pm 1.3$ stellar encounters per Myr within one pc of the Sun. We examine the ability of two future missions, FAME and GAIA, to extend the search for past and future stellar encounters with the Sun.
\end{abstract}

Key words. comets: general - stars: general - stars: kinematics - Galaxy: general

\section{Introduction}

Comets in the Oort cloud evolve dynamically under the influence of external perturbers. Their orbits are perturbed by random passing stars, by giant molecular clouds, and by the galactic gravitational field. In particular, the random motions of the stars and the Sun occasionally bring a star very close to the Sun. Close or penetrating passages through the Oort cloud can deflect large numbers of comets on to orbits that enter the planetary region (Hills 1981; Weissman 1996), thus triggering what are known as comet showers. Some terrestial impact craters and stratigraphic records of impact and extinction events on Earth (Hut et al. 1987), as well as geochemical evidence (Farley et al. 1998), suggest that such showers may have occurred in the past. The determination of the frequency of stellar encounters with the Sun is the starting point in the investigation of the role played by external perturbers, over the history of the solar system, on the dynamical evolution of the population of Oort cloud comets. Therefore, it

Send offprint requests to: J. García-Sánchez e-mail: joan.garcia@tin.it is important for our understanding of the solar system to answer questions such as how close and how often stellar encounters with the solar system occur, and what are the consequences for the dynamics of the cometary cloud.

The limited accuracy of pre-Hipparcos astrometric data, that is ground-based parallax and proper motion measurements, imposed a severe limitation on the accuracy of predictions of past or future close stellar passages. A significant improvement in the accuracy of astrometric data was achieved by the Hipparcos mission (ESA 1997). For instance, Jahreiss \& Wielen (1997) compared the best available ground-based trigonometric parallaxes of 1452 star systems with Hipparcos parallax values of the same systems. They found that the median standard error of the Hipparcos parallaxes is 1.15 milliarcsec (mas) compared to 8.8 mas for the best ground-based parallaxes, and that $40 \%$ of the ground-based parallaxes have errors exceeding 10 mas, compared with only $2 \%$ of the Hipparcos parallaxes. According to Hipparcos measurements, only $66 \%$ of the stars with ground-based distances closer than 25 pc are really within 25 pc. Using Hipparcos data, much better answers to the questions above can be obtained. 


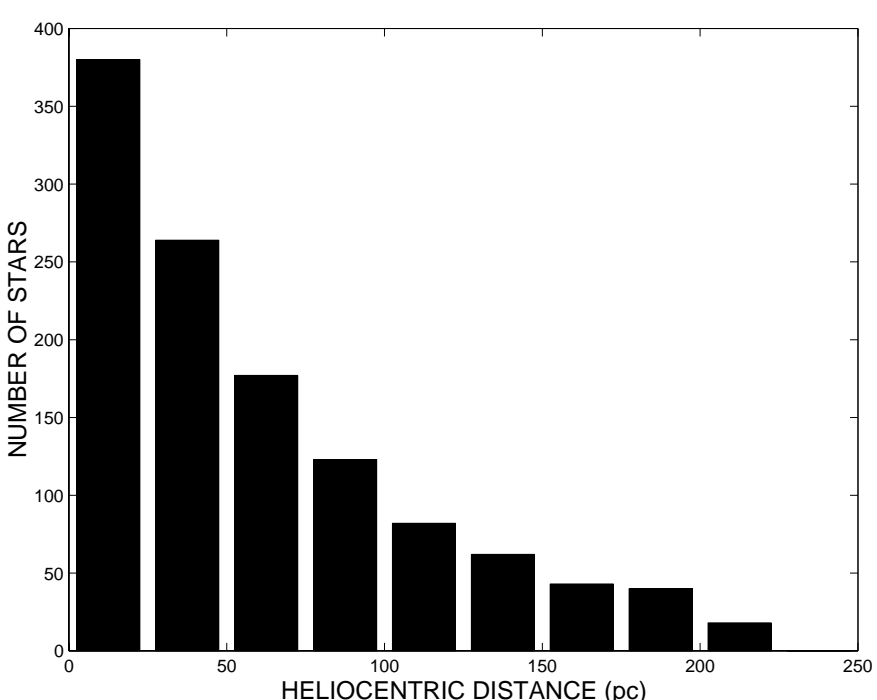

Fig. 1. Number of Hipparcos stars in our sample in each 25 pc bin as a function of distance from the Sun.

In García-Sánchez et al. (1997) we began the search for stars passing close to the Sun using Hipparcos data, assuming a simple linear motion model. In García-Sánchez et al. (1999, hereafter Paper I) we continued this search by integrating the motion of the candidate stars and the Sun in the local galactic potential. Based on radial velocity measurements from the literature as well as others from our own observations, we identified a few passing stars whose encounters with the solar system could potentially cause a perturbation of the Oort cloud. We performed dynamical simulations of cometary orbits using a Monte Carlo model to estimate if there is a significant increase in the long-period comet flux at the Earth's orbit caused by these potential perturbers. The strongest perturbation is for the future encounter with GL 710, though no substantial enhancement of the steady-state cometary flux would result from this passage.

In the present paper, we extend our search for close passages to more candidate stars by using new measurements of radial velocities, as well as considering several analytical expressions of the potential of the Galaxy to integrate the equations of motion. We study the limits of validity of our results, and how these limits may be expanded by new astrometric data from future space-based astrometric missions. In addition, we estimate the frequency of encounters with the Sun from the identified encounters taking into account the observational incompleteness of the Hipparcos data.

In Sect. 2 we present the data sources and the selection criteria for candidate stars used in the search for stellar encounters. In Sect. 3 we describe the equations of motion and the potential models of the Galaxy that will be used to compute the stellar galactic orbits. In Sect. 4 we discuss the range of values of the galactic parameters to be taken into account in the potential models, as well as the choice of the potential model that is the best suited to our study. The results of the predicted encounters with the solar system are presented in Sect. 5. In Sect. 6 we analyze different sources of uncertainty that may constrain this study and the valid time interval for agreement between potential models. In Sect. 7 we determine the frequency of encounters with the Sun using different methods. In Sect. 8 we asses the future impact of two space-based astrometric missions, GAIA and FAME. Finally, our conclusions are given in Sect. 9.

\section{The candidate stars}

Our astrometric data set consists of the right ascensions, $\alpha$, declinations, $\delta$, trigonometric parallaxes, $\pi$, and the proper motion components in right ascension, $\mu_{\alpha \star}=$ $\mu_{\alpha} \cos \delta$, and declination, $\mu_{\delta}$, of the stars contained in the Hipparcos Catalogue.

As in Paper I, in order to construct a sample of candidate stars that could have or could have had a passage close to the solar system, we selected stars from the Hipparcos Catalogue whose proper motion, combined with an assumed maximum velocity of $100 \mathrm{kms}^{-1}$, implied an impact parameter (closest approach distance) of $3 \mathrm{pc}$ or less. This velocity limit is several times the local stellar velocity dispersion, so that intrinsically high velocity stars are included. At that velocity this requirement means that stars whose proper motion in mas/yr is less than 0.06 times the square of the parallax in mas, are the best candidates to have approaches within $3 \mathrm{pc}$ from the Sun. We selected stars with parallax values greater than 4.5 mas because for smaller values the implied proper motion limit would be close to or below the Hipparcos measurement accuracy.

The impact parameter of $3 \mathrm{pc}$ allows inclusion of relatively distant passages of massive stars or star systems that might affect the cometary orbits. The net heliocentric velocity impulse gained by an Oort cloud comet as a result of a stellar passage is proportional to $M_{*} v_{*}^{-1} D_{\text {ca }}^{-2}$ (Rickman 1976), where $M_{*}, v_{*}$ and $D_{\text {ca }}$ are the mass, encounter velocity and closest approach distance, respectively, of the passing star or star system. Very close passages are expected to be the most likely to significantly perturb the Oort cloud. However, perturbations could also be possible for somewhat more distant ones, depending on how long the encounter lasts and how massive the stars or multiple star systems encountered are.

According to the above criteria, and after elimination of a few stars with unreliable astrometric values, we found a total of 1189 candidates that satisfied our search criteria. The distribution of the candidate stars with distance is shown in Fig. 1. The number of sample stars decreases with distance, with $80 \%$ of the stars within a heliocentric distance of 100 pc, and only $20 \%$ between 100 and 225 pc.

We searched the literature for published radial velocity measurements for the selected stars. We also made radial velocity measurements using the Center for Astrophysics (CfA) digital speedometers (Latham 1985, 1992) for some of the stars, as part of an observational program to measure radial velocities of candidate stars with no previous 


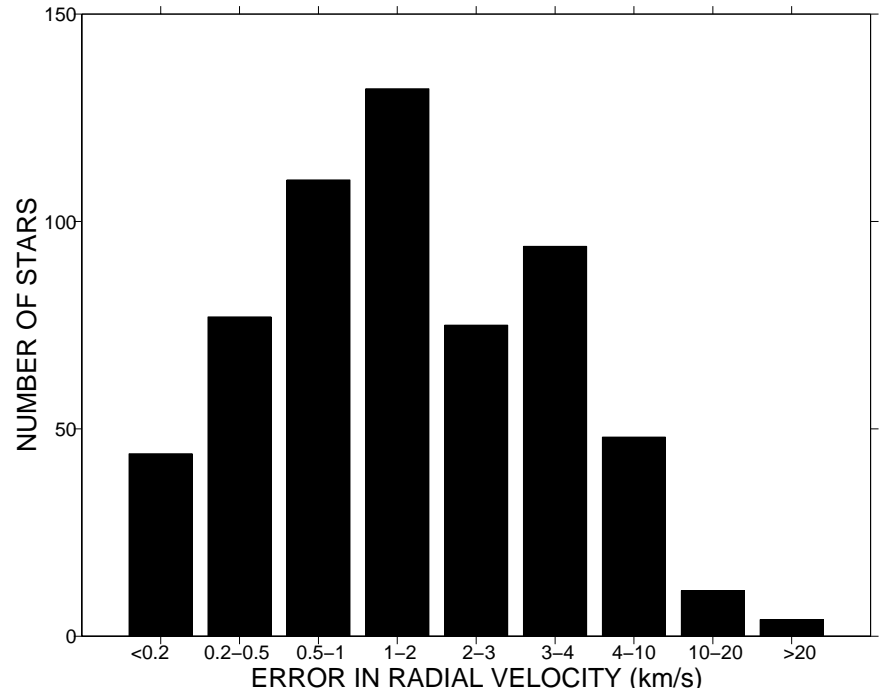

Fig. 2. Distribution of the radial velocity errors for the 595 candidate stars with measurements. Note that different bin widths are used.

measurements (see Paper I for details). We found values for a total of 595 of the 1189 candidate stars, 518 in the literature plus 77 observed at the CfA, about $50 \%$ of the total number selected. We plot the distribution of errors in the radial velocities of these 595 stars in Fig. 2.

\section{Potential models}

In order to determine the stellar encounters with the solar system, we consider the three-dimensional Newtonian equations of motion of a star with respect to the galactic center to compute the stellar trajectories under the galactic gravitational forces. Assuming a steady-state Galaxy with gravitational potential $\Phi$, these equations can be expressed in cylindrical coordinates $(R, \theta, z)$ centered on the galactic nucleus as (Mihalas \& Routly 1968)

$\ddot{R}-R \dot{\theta}^{2}=-\frac{\partial \Phi}{\partial R}$

$R^{2} \ddot{\theta}+2 R \dot{R} \dot{\theta}=-\frac{\partial \Phi}{\partial \theta}$

$\ddot{z}=-\frac{\partial \Phi}{\partial z}$

We will consider three models of the galactic potential that can be introduced in the equations of motion above. The first is a simple local potential model, which is based on the observed local features in the solar neighborhood. The second is the global potential model, and takes into account the large scale features of the Galaxy. Both the local and the global galactic potential models adopt an axisymmetric galactic potential, which implies that the partial derivatives of this potential with respect to $\theta$ are zero. This axisymmetry is broken when we consider the third model, the global model with the added perturbative contribution of the spiral arms of the Galaxy.

\subsection{Local potential model}

We assume that for stars not far from the galactic plane (small values of $z / R$ ), the galactic force can be expressed in terms of two components: a radial force $K_{R}$ that governs the motion in the galactic plane, and a vertical force $K_{z}$ that depends on the local mass density. Expanding the galactic force field in the plane to first order around the Sun's galactocentric distance $R_{\odot}$, the following empirical expression for the radial force $K_{R}$ (e.g., Trumpler \& Weaver 1953) can be derived:

$K_{R} \simeq K_{R_{\odot}}+\left(\frac{\mathrm{d} K_{R}}{\mathrm{~d} R}\right)_{\odot}\left(R-R_{\odot}\right)$.

Introducing $K_{R}=-\partial \Phi / \partial R$, the corresponding potential expression can be derived:

$\frac{\partial \Phi}{\partial R} \simeq\left(\frac{\partial \Phi}{\partial R}\right)_{\odot}+\left[\frac{\mathrm{d}}{\mathrm{d} R}\left(\frac{\partial \Phi}{\partial R}\right)\right]_{\odot}\left(R-R_{\odot}\right)$.

Introducing the expressions

$\left(\frac{\partial \Phi}{\partial R}\right)_{\odot}=\frac{\Theta_{\odot}^{2}}{R_{\odot}}$

and

$\left[\frac{\mathrm{d}}{\mathrm{d} R}\left(\frac{\partial \Phi}{\partial R}\right)\right]_{\odot}=2 \frac{\Theta_{\odot}}{R_{\odot}}\left(\frac{\mathrm{d} \Theta}{\mathrm{d} R}\right)_{\odot}-\frac{\Theta_{\odot}^{2}}{R_{\odot}^{2}}$

into Eq. (5), with $\Theta_{\odot}=\Omega_{\odot} R_{\odot}$ being the circular velocity at the Sun's position, we obtain

$\frac{\partial \Phi}{\partial R}=\Omega_{\odot}^{2} R_{\odot}+\left(2 \Omega_{\odot}\left(\frac{\mathrm{d} \Theta}{\mathrm{d} R}\right)_{\odot}-\Omega_{\odot}^{2}\right)\left(R-R_{\odot}\right)$.

For the perpendicular motion relative to the galactic plane, we can use Poisson's equation to derive $\partial \Phi / \partial z$. Poisson's equation, to first order, is

$4 \pi G \rho_{\odot}=-\frac{\partial K_{\mathrm{z}}}{\partial z}$

where $\rho_{\odot}$ is the mass density in the solar neighborhood. The term neglected in this equation, $2\left(A^{2}-B^{2}\right)$, where $A$ and $B$ are the Oort constants, is zero for a flat galactic rotation curve and small for other rotation curves. Since $K_{z}=-\partial \Phi / \partial z$, we can write:

$\frac{\partial \Phi}{\partial z}=4 \pi G \rho_{\odot} z$

\subsection{Global potential model}

Next we move to a more global view of the Galaxy, and consider a galactic mass model based on the large scale features of the Galaxy. The structure of the Galaxy can be modeled as the contributions of three components - a bulge, a disk and a halo - in order to reproduce the global galactic gravitational potential. 
In the present case, the total axisymmetric galactic potential can be expressed as the sum of the contributions of each component:

$\Phi \equiv \Phi_{\mathrm{gl}}=\Phi_{\mathrm{b}}+\Phi_{\mathrm{d}}+\Phi_{\mathrm{h}}$

where $\Phi_{\mathrm{b}}, \Phi_{\mathrm{d}}$ and $\Phi_{\mathrm{h}}$ are the potentials generated by the bulge, disk and halo, respectively.

For the potential-density pair of the spherically symmetric bulge and halo components, we adopt Plummer's (1911) model which can be written as

$\Phi_{\mathrm{b}, \mathrm{h}}=-\frac{G M_{\mathrm{b}, \mathrm{h}}}{\sqrt{R^{2}+z^{2}+b_{\mathrm{b}, \mathrm{h}}^{2}}}$

whereas for the axisymmetric disk we adopt the Miyamoto \& Nagai (1975) form of Kuzmin's (1956) model:

$\Phi_{\mathrm{d}}=-\frac{G M_{\mathrm{d}}}{\sqrt{R^{2}+\left(a_{\mathrm{d}}+\sqrt{z^{2}+b_{\mathrm{d}}^{2}}\right)^{2}}}$.

In the above expressions $M_{\mathrm{b}}, M_{\mathrm{d}}$ and $M_{\mathrm{h}}$ are the mass of the bulge, disk and halo, respectively, $a_{\mathrm{d}}$ is the scale length of the disk, and $b_{\mathrm{b}}, b_{\mathrm{d}}$ and $b_{\mathrm{h}}$ are the scale heights of the bulge, disk and halo, respectively.

\subsection{Perturbative potential model}

We next consider the effect of a small-amplitude density perturbation of the galactic potential, such as the perturbing spiral gravitational potential superimposed on the axisymmetric galactic disk by the existence of spiral arms. We assume that the spiral arms are a rigidly rotating pattern on the galactic disk that sets up a local minimum in the gravitational field of the disk.

The potential $\Phi_{\mathrm{s}}$ associated with this perturbation can in general be described as a superposition of elementary waves of the form (see, e.g., Yuan 1969a,b; Bertin \& Lin 1996):

$\Phi_{\mathrm{s}}=\Re \mathrm{e}\left\{\Upsilon(R) \mathrm{e}^{i(\omega t-m \theta)}\right\}$

where $m$ is the number of spiral arms. The parameter $\omega$ is related to the angular speed at which the spiral pattern rotates about the galactic center, $\Omega_{\mathrm{p}}$, through $\omega=m \Omega_{\mathrm{p}}$. The function $\Upsilon(R)$ is

$\Upsilon(R)=A(R) \mathrm{e}^{i \Psi(R)}$

where $A(R)$ is the amplitude and $\Psi(R)$ is the phase term. The amplitude $A(R)$ is a slowly varying function of $R$, and for our purposes is taken to be

$A=\frac{F R_{\odot}^{2} \Omega_{\odot}^{2} \tan \gamma}{m}$

with $F$ being the ratio of the radial component of the spiral field to the symmetrical field, and $\gamma$ the pitch angle of the spiral arm given by

$\tan \gamma=\frac{m}{2 \pi} \ln \left(1+\frac{\Delta R}{R_{\mathrm{a}}}\right)$.
In the expression above, $R_{\mathrm{a}}$ is the location of the center of the spiral arm in the direction of the galactic center and $\Delta R$ is the separation between arms. The phase term $\Psi(R)$ is

$\Psi(R)=\Psi_{\odot}-\frac{m}{\tan \gamma} \ln \left(\frac{R}{R_{\odot}}\right)$

where $\Psi_{\odot}$ is a constant that can be determined from the condition of minimum of the potential (that is, for $t=0$, $\left.\theta=0^{\circ}, \Psi(R)=\pi\right)$.

Following Yuan (1969b), we can express Eq. (14) as

$\Phi_{\mathrm{s}}=A \cos \left(m\left(\Omega_{\mathrm{p}} t-\theta\right)+\Psi(R)\right)$.

To obtain the total potential $\Phi$ used in the equations of motion, $\Phi_{\mathrm{s}}$ is added to the axisymmetric global potential, denoted as $\Phi_{\mathrm{gl}}$ and given by (11). Thus, the total potential is given as

$\Phi=\Phi_{\mathrm{gl}}+\Phi_{\mathrm{s}}$

where the partial derivatives with respect to $R, \theta$ and $z$ are

$\frac{\partial \Phi}{\partial R}=\frac{\partial \Phi_{\mathrm{gl}}}{\partial R}+\frac{\partial \Phi_{\mathrm{s}}}{\partial R}$

$\frac{\partial \Phi}{\partial \theta}=\frac{\partial \Phi_{\mathrm{gl}}}{\partial \theta}+\frac{\partial \Phi_{\mathrm{s}}}{\partial \theta}=\frac{\partial \Phi_{\mathrm{s}}}{\partial \theta}$

$\frac{\partial \Phi}{\partial z}=\frac{\partial \Phi_{\mathrm{gl}}}{\partial z}+\frac{\partial \Phi_{\mathrm{s}}}{\partial z}=\frac{\partial \Phi_{\mathrm{gl}}}{\partial z}$.

\section{Galactic parameters and choice of potential model}

We discuss here the values of the parameters to be used in the equations of motion described above. Our aim is to construct a set of plausible and self-consistent values of the galactic parameters, according to observational constraints in the literature, from which the stellar passages can then be determined.

\subsection{Solar and galactic parameters in the local potential model}

For the Sun's velocity with respect to the Local Standard of Rest (LSR), we adopt the values of $9.3 \pm 0.8 \mathrm{~km} \mathrm{~s}^{-1}$ in the direction of the galactic center, $11.2 \pm 0.7 \mathrm{~km} \mathrm{~s}^{-1}$ in the direction of galactic rotation, and $7.6 \pm$ $0.6 \mathrm{~km} \mathrm{~s}^{-1}$ towards the north galactic pole, as reported by Feast \& Whitelock (1997), and very similar to Delhaye's (1965) classical values of $(9,12,7) \mathrm{km} \mathrm{s}^{-1}$. An accurate value for the Sun's vertical height above the plane, $z_{\odot}$, is not well established. Past studies report values of $z_{\odot}$ ranging from about 10 to $42 \mathrm{pc}$, depending on the method used (see Humphreys \& Larsen 1995 for a review). Reed (1997) estimated that $z_{\odot}$ is likely not less than $\sim 6 \mathrm{pc}$ and no more than $\sim 13 \mathrm{pc}$ above the galactic midplane based on OB star counts. Hipparcos studies also report low heights, $z_{\odot} \simeq 8 \pm 4 \mathrm{pc}$ according to Holmberg et al. (1997) 
Table 1. Values of the solar and galactic parameters adopted.

\begin{tabular}{ll}
\hline Sun's motion & $(9.3,11.2,7.6) \mathrm{km} \mathrm{s}^{-1}$ \\
Sun's height & $z_{\odot}=10 \mathrm{pc}$ \\
Galactocentric distance & $R_{\odot}=7.5-8.5 \mathrm{kpc}$ \\
Local mass density & $\rho_{\odot}=0.076-0.15 \mathrm{M}_{\odot} \mathrm{pc}^{-3}$ \\
Local angular velocity & $\Omega_{\odot}=27.19 \mathrm{~km} \mathrm{~s}^{-1} \mathrm{kpc}^{-1}$ \\
& $(\mathrm{~d} \Theta / \mathrm{d} R)_{\odot}=-2.4 \mathrm{~km} \mathrm{~s}^{-1} \mathrm{kpc}^{-1}$ \\
Rotation constants & $A=14.82 \mathrm{~km} \mathrm{~s}^{-1} \mathrm{kpc}^{-1}$ \\
& $B=-12.37 \mathrm{~km} \mathrm{~s}^{-1} \mathrm{kpc}^{-1}$ \\
\hline
\end{tabular}

and $z_{\odot}=9 \pm 4 \mathrm{pc}$ according to Pham (1997). We adopt a rounded-off value of $z_{\odot}=10 \mathrm{pc}$. We will show later that the use of other plausible values of $z_{\odot}$ does not significantly affect our calculations. The IAU standard value for the Sun's galactocentric distance is $R_{\odot}=8.5 \pm 1.1 \mathrm{kpc}$ (Kerr \& Lynden-Bell 1986). However, more recent studies show a trend towards smaller values of $R_{\odot}$. Reid (1993) examined this question and found a best value of $R_{\odot}=$ $8.0 \pm 0.5 \mathrm{kpc}$. Feast \& Whitelock (1997), in a study of the local galactic kinematics based on Hipparcos proper motion measurements of Cepheids, derived $R_{\odot}=8.5 \pm$ $0.5 \mathrm{kpc}$. In constrast, Olling \& Merrifield (1998) also studied the rotation curve of the Galaxy and derived $R_{\odot}=$ $7.1 \pm 0.5 \mathrm{kpc}$, a value that they claim to be consistent with the kinematic study by Feast \& Whitelock (1997). In our study we adopt the range $R_{\odot}=7.5-8.5 \mathrm{kpc}$ as the most reliable values.

By "galactic parameters", we refer here to those that characterize the amount of matter and the rotation of the Galaxy in the solar neighborhood. Several studies have been carried out to determine the local mass density $\rho_{\odot}$ dynamically, leading to conflicting results. Whereas some authors claimed the presence of disk dark matter greater than $50 \%$ of the observed matter (Bahcall 1984a,b; Bahcall et al. 1992), others estimated a much lower value or even zero disk dark matter (Bienaymé et al. 1987; Kuijken \& Gilmore 1989). Work based on Hipparcos data seems to support a low value of the local dynamical mass density; Crézé et al. (1998) found $\rho_{\odot}=0.076 \pm 0.015 M_{\odot} \mathrm{pc}^{-3}$, Holmberg \& Flynn $(2000)$ found $\rho_{\odot}=0.102 \pm 0.01 M_{\odot} \mathrm{pc}^{-3}$, and Pham (1997) found $\rho_{\odot}=0.11 \pm 0.01 M_{\odot} \mathrm{pc}^{-3}$. The differences in the Hipparcos results are mainly due to the methods applied. We adopt the range $\rho_{\odot}=0.076-0.15 M_{\odot} \mathrm{pc}^{-3}$ in our study.

Based on Hipparcos proper motions of Cepheids, Feast $\&$ Whitelock (1997) derived Oort constants of $A=14.82 \pm$ $0.84 \mathrm{~km} \mathrm{~s}^{-1} \mathrm{kpc}^{-1}$ and $B=-12.37 \pm 0.64 \mathrm{~km} \mathrm{~s}^{-1} \mathrm{kpc}^{-1}$. These results are essentially independent of the value of $R_{\odot}$ used. Feast \& Whitelock also derived an angular velocity of circular rotation of $\Omega_{\odot}=27.19 \pm$ $0.87 \mathrm{~km} \mathrm{~s}^{-1} \mathrm{kpc}^{-1}$, in very good agreement with the value of $27.2 \pm 1.7 \mathrm{~km} \mathrm{~s}^{-1} \mathrm{kpc}^{-1}$ derived by Reid et al. (1999), and a slowly declining galactic rotation curve at $R_{\odot}$, given by $(\mathrm{d} \Theta / \mathrm{d} R)_{\odot}=-2.4 \pm 1.2 \mathrm{~km} \mathrm{~s}^{-1} \mathrm{kpc}^{-1}$. We adopt these values in our calculations.
Table 2. Adopted constants $R_{\odot}$ and $\Theta_{\odot}$, model constants and computed local parameters of the model of Dauphole \& Colin (1995).

\begin{tabular}{ll}
\hline Sun's Galactocentric dist. & $R_{\odot}=8.0 \mathrm{kpc}$ \\
Local circular velocity & $\Theta_{\odot}=225 \mathrm{~km} \mathrm{~s}^{-1}$ \\
Local angular velocity & $\Omega_{\odot}=28.125 \mathrm{~km} \mathrm{~s}^{-1} \mathrm{kpc}^{-1}$ \\
Bulge constants & $M_{\mathrm{b}}=1.3955 \times 10^{10} M_{\odot}$ \\
& $b_{\mathrm{b}}=0.35 \mathrm{kpc}$ \\
Disk constants & $M_{\mathrm{d}}=7.9080 \times 10^{10} M_{\odot}$ \\
& $a_{\mathrm{d}}=3.55 \mathrm{kpc}$ \\
& $b_{\mathrm{d}}=0.25 \mathrm{kpc}$ \\
Halo constants & $M_{\mathrm{h}}=6.9766 \times 10^{11} M_{\odot}$ \\
& $b_{\mathrm{h}}=24.0 \mathrm{kpc}$ \\
Local mass density & $\rho_{\odot}=0.143 M_{\odot} \mathrm{pc}^{-3}$ \\
Rotation constants & $A=14.25 \mathrm{~km} \mathrm{~s}^{-1} \mathrm{kpc}^{-1}$ \\
& $B=-13.89 \mathrm{~km} \mathrm{~s}^{-1} \mathrm{kpc}^{-1}$ \\
\hline
\end{tabular}

The selected values of the parameters are all listed in Table 1.

\subsection{Global potential model parameters}

We cannot adopt the values listed in Table 1 directly for the global potential model. The reason is that we need additional parameters to describe the global potential model, for example, the masses of the different components (bulge, disk and halo) that contribute to the potential. The values of these additional parameters are determined from the best global fit to the Galaxy according to certain observational constraints. Values of these additional parameters can be found in the literature, but if we adopt them we should also adopt the values of the other model parameters derived from the same fit. Otherwise, our model would not be internally consistent.

By adopting a set of parameters for the global potential model from the literature we demand, however, that their values be in reasonable agreement with those listed in Table 1. We adopt the parameters derived by Dauphole \& Colin (1995) for a global potential model, which fulfill the above requirement. Their model consists of an axisymmetric galactic potential generated by the contributions of a spherical central bulge, a disk and an extended spherical halo. The values of the parameters were derived by Dauphole \& Colin from the classical observational constraints (rotation curve, local perpendicular force, local density and Oort constants), but in addition these authors also used the dynamics of the galactic globular clusters as a constraint on the model parameters.

The values derived by Dauphole \& Colin are listed in Table 2. The Sun's galactocentric distance $R_{\odot}$ and local circular velocity $\Theta_{\odot}$, as adopted by Dauphole \& Colin, are $R_{\odot}=8.0 \mathrm{kpc}$ and $\Theta_{\odot}=225 \mathrm{~km} \mathrm{~s}^{-1}$, respectively. The total mass of the Galaxy derived from this model is $7.9 \times 10^{11} M_{\odot}$. For the Sun's motion we adopt the velocity components $(9.3,11.2,7.6) \mathrm{km} \mathrm{s}^{-1}$, and for the Sun's height above the galactic midplane we adopt the value $z_{\odot}=10 \mathrm{pc}$, as discussed above. 


\subsection{Spiral arm parameters}

There is not a single, accepted picture of the global spiral structure of the Galaxy. Models differ in the number of spiral arms, $m$, the value of the pitch angle, $\gamma$, of the arms, and the value of the pattern speed of rotation $\Omega_{\mathrm{p}}$. Proposed global fits to observations of the Galaxy include either two or four arms, with pitch angles ranging from $\sim 5^{\circ}$ to $\sim 27^{\circ}$ (see Elmegreen 1985 for a review and references to these observations). For instance, Henderson (1977) and Blitz et al. (1983) obtained a four-armed pattern from HI and CO data, whereas Simonson (1976) obtained a two-armed inner structure with two additional outer arms. The use of the density-wave theory of spiral arms obtains two-armed spirals for the study of young objects in the solar neighborhood. From optical HII regions, Georgelin \& Georgelin (1976) delineated four arm segments, though Bash (1981) showed that a two-armed model could also account for the HII regions used by Georgelin \& Georgelin. With regard to the spiral pattern speed, results from several studies basically cluster around two different ranges of values, $\Omega_{\mathrm{p}} \simeq 11-14 \mathrm{~km} \mathrm{~s}^{-1} \mathrm{kpc}^{-1}$ (Lin et al. 1969; Yuan 1969a,b; Gordon 1978) and $\Omega_{\mathrm{p}} \simeq$ 20-28 km s${ }^{-1} \mathrm{kpc}^{-1}$ (Crézé \& Mennessier 1973; Nelson \& Matsuda 1977; Avedisova 1989; Amaral \& Lépine 1997; Mishurov \& Zenina 1999). The differences between these values depends mainly on the techniques used to estimate the pattern speed. Models implying that the spiral waves travel outwards from the center of the Galaxy result in larger values of $\Omega_{\mathrm{p}}$, whereas those implying the opposite propagation direction obtain lower pattern speed values.

According to the density-wave theory of the galactic spiral arms, the density wave pattern propagates around the Galaxy with a pattern speed $\Omega_{\mathrm{p}}$ that can only extend over the part of the galactic disk for which (Lin et al. 1969)

$\Omega-\frac{\kappa}{m}<\Omega_{\mathrm{p}}<\Omega+\frac{\kappa}{m}$

where $\Omega$ is the angular velocity of rotation at a distance $R, \kappa$ is the epicyclic frequency at this distance, and $m$ is the number of spiral arms. The epicyclic frequency is defined as

$\kappa^{2}=4 \Omega^{2}\left(1+\frac{R}{2 \Omega} \frac{\mathrm{d} \Omega}{\mathrm{d} R}\right)$.

The values of $\Omega-\kappa / m$ and $\Omega+\kappa / m$ are the inner and outer Lindblad resonances, respectively. At resonance locations, energy is dissipated and can lead to damping of spiral waves. In addition, a corotation resonance can occur if $\Omega_{\mathrm{p}}=\Omega$ for some value of $R$. At the corotation circle, the wave can be amplified through over-reflection (e.g., Lin \& Bertin 1985). The density-wave theory works best at the zone between the inner Lindblad resonance and the corotation resonance.

In our model of the Galaxy we adopted the parameters by Dauphole \& Colin (1995). In order to study the range of values of $\Omega_{\mathrm{p}}$ permitted by condition (24) and consistent with these parameters, we first derive the rotation curve

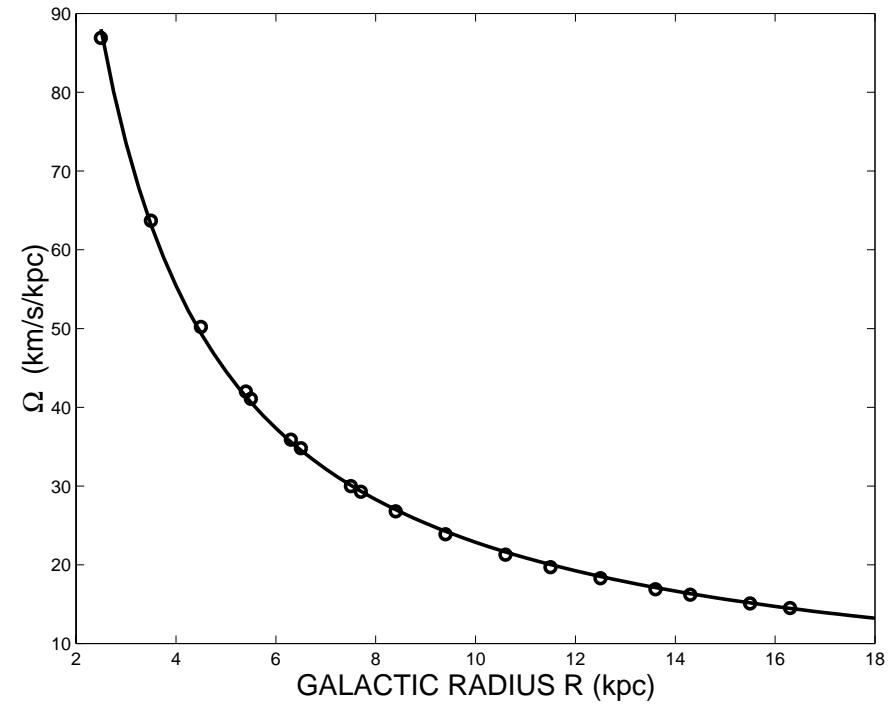

Fig. 3. Rotation curve of the Galaxy as a function of distance $R$ from the galactic center. The circles represent the values listed by Dauphole \& Colin (1995), derived from their model.

of the Galaxy $\Omega(R)$ by means of a least-squares linear fit to the values tabulated by Dauphole \& Colin (1995). From this curve we then determine the allowed $\Omega_{\mathrm{p}}$. The fit to the rotation curve values listed by Dauphole \& Colin is

$\left(\frac{\Omega}{\Omega_{\odot}}\right)=0.965\left(\frac{R_{\odot}}{R}\right)+0.041$

where $\Omega_{\odot}$ and $R_{\odot}$ are the values listed in Table 2 . Figure 3 shows the fitted curve.

The epicyclic frequency $\kappa$ as a function of distance $R$ can be derived by introducing expression (26) and its derivative with respect to $R$ into Eq. (25). Therefore, both $\Omega(R)$ and $\kappa(R)$ can be used to determine the range of values of the pattern speed compatible with condition (24).

We plot the rotation curve of the Galaxy $\Omega$ given by Eq. (26) in Fig. 4, and also the inner and outer Lindblad resonances (the values of $\Omega \pm \kappa / m$ ) for $m=2$ and $m=$ 4 spiral arms. The Lindblad resonances determine the boundary of the region where the spiral structure can exist, the region enclosed between the curves $\Omega+\kappa / m$ and $\Omega-\kappa / m$ in the plot.

For the high range of $\Omega_{\mathrm{p}}$ we consider here the two extreme values above, $\Omega_{\mathrm{p}}=20.0 \mathrm{~km} \mathrm{~s}^{-1} \mathrm{kpc}^{-1}$ and $\Omega_{\mathrm{p}}=$ $28 \mathrm{~km} \mathrm{~s}^{-1} \mathrm{kpc}^{-1}$. For the low range we consider the value of $\Omega_{\mathrm{p}}=13.5 \mathrm{~km} \mathrm{~s}^{-1} \mathrm{kpc}^{-1}$ (Yuan 1969a). These three values are represented in Fig. 4 by the three horizontal lines. This plot suggests a preference for the two-armed over the four-armed spiral structure for low values of $\Omega_{\mathrm{p}}$. The two-armed pattern can extend over a larger region of the Galaxy. For $\Omega_{\mathrm{p}}=13.5 \mathrm{~km} \mathrm{~s}^{-1} \mathrm{kpc}^{-1}$, the four-armed spiral structure is limited to exist only in the outer parts of the Galaxy.

Assuming a solar galactocentric distance of $R_{\odot}=$ $8.0 \mathrm{kpc}$, the Sagittarius arm is located at $R_{\mathrm{a}}=6.5 \mathrm{kpc}$, with the arm separation (distance from the Sagittarius arm to the Perseus arm) being $\Delta R \simeq 3 \mathrm{kpc}$ (Vallée 1995). 


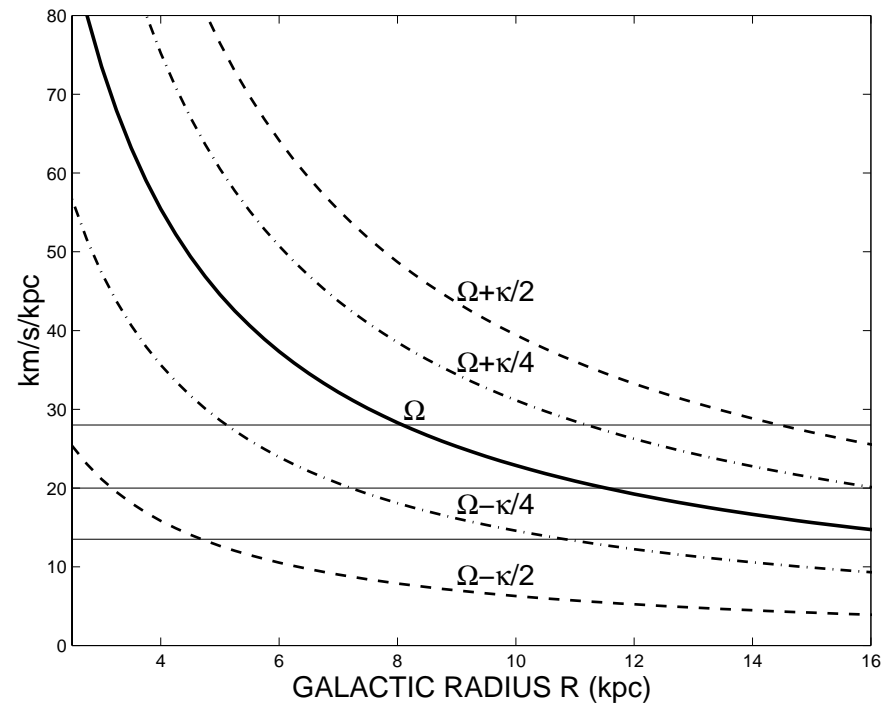

Fig. 4. Rotation curve of the Galaxy, $\Omega$ (heavy line), and $\Omega \pm \kappa / m$ curves for $m=2$ and $m=4$, as a function of galactic radius. The case $m=2$ is plotted with a dashed line whereas the case $m=4$ is plotted with a dot-dashed line. The three horizontal lines indicate where $\Omega_{\mathrm{p}}$ is $13.5 \mathrm{~km} \mathrm{~s}^{-1} \mathrm{kpc}^{-1}$, $20.0 \mathrm{~km} \mathrm{~s}^{-1} \mathrm{kpc}^{-1}$ and $28.0 \mathrm{~km} \mathrm{~s}^{-1} \mathrm{kpc}^{-1}$, from bottom to top, respectively.

A pure four-armed spiral structure can account for the inner galactic arms only when higher values of $\Omega_{\mathrm{p}}$ are considered. From Eq. (24) we find that for the galactocentric radius $R_{\odot}=8.0 \mathrm{kpc}$ the pattern speed of rotation should be $\Omega_{\mathrm{p}}>8.4 \mathrm{~km} \mathrm{~s}^{-1} \mathrm{kpc}^{-1}$ for a two-armed spiral and $\Omega_{\mathrm{p}}>18.3 \mathrm{~km} \mathrm{~s}^{-1} \mathrm{kpc}^{-1}$ for a four-armed spiral. Thus, a four-armed spiral pattern can exist in the inner galactic disk only with a pattern speed greater than $\sim 20 \mathrm{~km} \mathrm{~s}^{-1} \mathrm{kpc}^{-1}$. Moreover, in our case a four-armed structure can account for the existence of the Sagittarius arm only if pattern speeds greater than $\sim 25 \mathrm{~km} \mathrm{~s}^{-1} \mathrm{kpc}^{-1}$ are considered. However, if $25.0 \mathrm{~km} \mathrm{~s}^{-1} \mathrm{kpc}^{-1}<\Omega_{\mathrm{p}}<$ $28.0 \mathrm{~km} \mathrm{~s}^{-1} \mathrm{kpc}^{-1}$, the corotation resonance would be located at a galactic radius between that of the Perseus arm and the Sun's galactocentric distance.

Since a pure four-armed structure is constrained to a much smaller region of the Galaxy than the two-armed structure, and also to avoid corotation resonance at such a galactocentric distance, we adopt the two-armed structure for the spiral potential model. For the sake of comparison, we consider two values of the pattern speed $\Omega_{\mathrm{p}}, 13.5$ and $20.0 \mathrm{~km} \mathrm{~s}^{-1} \mathrm{kpc}^{-1}$. We list the values for the spiral potential perturbation in Table 3 . We adopt the value $F=$ 0.05 (Yuan 1969b) for the ratio of the radial component of the spiral field.

\subsection{Choice of potential model}

The local potential model assumes that for the motion of stars not far away from the galactic plane, the vertical $z$-force, which essentially depends on the local mass density $\rho_{\odot}$, can be decoupled from the $R$-force parallel to
Table 3. Parameters adopted for the spiral structure of the Galaxy.

\begin{tabular}{lc} 
Number of spiral arms, $m$ & 2 \\
Pitch angle, $\gamma$ (degrees) & $6.9^{\circ}$ \\
Spiral pattern speed, $\Omega_{\mathrm{p}}\left(\mathrm{km} \mathrm{s}^{-1} \mathrm{kpc}^{-1}\right)$ & $13.5,20.0$ \\
Ratio spiral to symmetrical field, $F$ & 0.05 \\
Location of Sagittarius arm, $R_{\mathrm{a}}(\mathrm{kpc})$ & 6.5 \\
Distance Sagittarius to Perseus arm, $\Delta R(\mathrm{pc})$ & 3.0 \\
\hline
\end{tabular}

the galactic plane. The equation of vertical motion $z$ results in a harmonic motion above and below the galactic midplane, which is valid only for stars with small amplitudes of oscillation, $z_{\max }$ of less than $\sim 300$ pc (Binney \& Tremaine 1987). For larger distances from the galactic midplane the $K_{z}$ force drops below its linear value and the harmonic approximation does not work as well.

To estimate the validity of this approximation for our candidate stars, we calculated the amplitude $z_{\max }$ of the vertical motion of the stars for which we have radial velocity measurements, assuming a nominal value of $\rho_{\odot}=$ $0.1 M_{\odot} \mathrm{pc}^{-3}$. Ninety per cent of the candidate stars reach a maximum distance from the galactic plane of less than $300 \mathrm{pc}$, and about one half of the stars have vertical oscillation amplitudes within 100 pc of the plane. Only $10 \%$ of the stars reach distances greater than $300 \mathrm{pc}$ from the midplane, up to $\sim 1 \mathrm{kpc}$. For this $10 \%$ of stars the departure from the harmonic motion approximation may be significant. Therefore, the application of the local potential model is restricted to the case of candidate stars whose orbits are relatively close to the midplane. However, when the coupling between vertical and radial galactic forces can be neglected, this model provides a good description of the galactic potential in the solar neighborhood. It also has the advantage of allowing the use of well determined local parameters, so we can easily test the effect of varying any of these parameters on the stellar orbits.

On the other hand, dynamical mass models of the Galaxy, represented in our case by the global potential model, attempt to fit the observational parameters directly related to the force field of the Galaxy. The basic fit is to the galactic rotation curve, which contains information about the radial force field in the plane at various radial distances, and to the perpendicular force as a function of vertical height. Models thus constructed provide a description of the potential throughout the Galaxy. Therefore, the global potential model is preferred to the local potential one because its validity is not restricted to any particular region of the Galaxy or to a certain fraction of our candidate stars, contrary to what happens with the local potential model.

The global potential model parameters are adjusted to optimize the agreement between the kinematic predictions and the observational constraints of the galactic features. However, this large-scale adjustment results in a smoothed potential that might not fit local irregularities. In this case, the introduction of additional contributions from other components may be required, and the 
axisymmetry of the global potential model will be broken. The strongest perturbation on the smoothed potential model comes from the potential generated by the spiral arms of the Galaxy. The use of a perturbative potential together with the global potential model would thus represent an improvement in the description of the large-scale potential of the Galaxy. However, as discussed above, the determination of the global spiral structure of the Galaxy is problematic, since an accurate description of the spiral arm features is not well established, neither qualitatively nor quantitatively. We might also consider the contribution of a galactic central bar as a perturbation of the global potential. Dehnen (2000) performed numerical simulations of the velocity distribution adopting a nearly flat rotation curve and a rotating central bar, and found a signature in the velocity distribution caused by the outer Lindblad resonance of the bar. However, the values of parameters describing the central bar, found throughout the literature and based on different studies, such as the axis ratios, the orientation of the bar, and the pattern speed, are still a matter of debate (see, e.g., Gerhard 1999 for a review). Also, Asiain (1998) performed an analysis with a sample of solar neighborhood stars and showed that the perturbation of the bar on the galactic potential is much smaller than that of the spiral arms. He further showed that the effect of the bar on the galactic orbits of solar neighborhood stars can be neglected, at least within some tens of Myr from the present time. The times of closest passage determined with our integrations of galactic orbits (see next section) are relatively short, less than $\sim 10 \mathrm{Myr}$, a time interval for which the effect of the central bar on the star's trajectory is not significant.

We adopt the global potential model as the most reliable model to determine the stellar galactic orbits of our candidate stars. However, we will also use the local and the spiral potential models to study the limits of the model adopted.

\section{Determination of stellar encounters}

We computed the galactic orbital motion of the Sun and the 595 candidate stars with measured radial velocities using the global potential model. The adopted galactic parameters are those derived by the model of Dauphole \& Colin (1995), listed in Table 2. The integrations were performed for a time $\pm 100 \mathrm{Myr}$ from the present time, using a fourth-order Runge-Kutta integrator. The 100 Myr interval of time is large enough to allow all our candidate stars to encounter the solar system at their closest approach distance. From the computed stellar and solar galactic orbits, we determined the parameters of the closest approach to the Sun for each candidate star.

We list the encounters with the candidate stars in Table 4 , in order of increasing miss distance $D_{\text {ca }}$, for those 156 stars with a miss distance within 5 pc of the Sun. The predicted passages are contained in a time interval of \pm 10 Myr from the present, with most of them, $\sim 85 \%$, occurring within \pm 3 Myr. Eighty seven of the candidate stars listed are predicted to encounter the solar system in the future, compared with 69 candidate stars having their closest approach in the past. The past/future ratio is just over one sigma different than what would be expected from random statistics.

We estimated the effect of a one sigma variation in the astrometric parameters used (parallax, proper motion in right ascension and declination, and radial velocity) on the predicted encounter parameters $D_{\text {ca }}$ and $T_{\text {ca }}$. We neglect the uncertainties in the position coordinates since they are very small. This estimate is given in Table 4 as $\Delta D_{\text {ca }}$ and $\Delta T_{\text {ca. }}$. The values of $\Delta D_{\text {ca }}$ and $\Delta T_{\text {ca }}$ were calculated as the root sum square of the changes in $D_{\text {ca }}$ and $T_{\text {ca }}$ due to individual one sigma increases in the parallaxes, proper motions and radial velocities of the candidate stars

$$
\begin{aligned}
& \Delta D_{\mathrm{ca}}=\sqrt{\sum_{i=1}^{4}\left(\Delta D_{i}\right)^{2}} \\
& \Delta T_{\mathrm{ca}}=\sqrt{\sum_{i=1}^{4}\left(\Delta T_{i}\right)^{2}}
\end{aligned}
$$

where the index $i$ denotes the four astrometric parameters used in the calculation. The radial velocities and their uncertainties are also given in Table 4 . We must caution that, in some cases, the astrometric and radial velocity errors might not be a reliable estimation of the true errors because of the long-period binaries not yet recognized.

The miss distance versus time of past (negative times) or future (positive times) encounters for the 156 passages within 5 pc are shown in Fig. 5. The shaded area represents the Oort cloud with a radius of $\sim 10^{5} \mathrm{AU}$ (Smoluchowski \& Torbett 1984; Antonov \& Latyshev 1972). The size of the data point for each star is proportional to the visual magnitude of the star at the predicted minimum distance. We note that passages at large times are dominated by stars with the largest apparent brightness at closest approach, which suggests an observational bias. Most of the stars encountering the Sun at large times from the present epoch could only have been observed by Hipparcos at present if they are intrinsically bright. We will examine this question of observational incompleteness in more detail later.

The spatial distribution of the closest approach points on the plane of the sky in galactic coordinates, for the 156 candidate stars with miss distances less than $5 \mathrm{pc}$, is shown in Fig. 6. The solar apex and antapex directions at the present time are also noted in the plot. We use different symbols to distinguish between past and future encounters. There appears to be some signs of non-randomness in the directions to the closest approach points, in particular the low density of points in some areas of the sky such as, for instance, near the solar antapex.

To estimate if there is any departure from randomness in the distribution of encounters induced by the solar motion direction, we plot the number of stars encountered within $5 \mathrm{pc}$ as a function of the angle between the solar 
Table 4. Predicted stellar encounters with the solar system for passages within a heliocentric distance of 5 pc. Negative times indicate passages in the past, whereas positive times indicate future passages.

\begin{tabular}{|c|c|c|c|c|c|c|c|}
\hline $\operatorname{HIP}^{a}$ & Name $^{b}$ & $D_{\mathrm{ca}}{ }^{c}$ & $\Delta D_{\mathrm{ca}}{ }^{d}$ & $T_{\mathrm{ca}}{ }^{e}$ & $\Delta T_{\mathrm{ca}}^{f}$ & $v_{\mathrm{r}}^{g}$ & $\sigma_{v_{\mathrm{r}}}^{h}$ \\
\hline 89825 & GL 710 & 0.337 & 0.177 & 1357.6 & 40.9 & -13.9 & 0.2 \\
\hline 85661 & HD 158576 & 0.938 & 0.705 & 1845.8 & 141.0 & -46.0 & 1.7 \\
\hline 70890 & Proxima Centauri & 0.954 & 0.036 & 26.7 & 0.2 & -21.7 & 1.8 \\
\hline 71683 & $\alpha$ Centauri A & 0.973 & 0.020 & 27.8 & 0.1 & -22.7 & 1.0 \\
\hline 71681 & $\alpha$ Centauri B & 0.975 & 0.020 & 27.7 & 0.2 & -22.7 & 1.0 \\
\hline 57544 & $\mathrm{AC}+793888$ & 1.007 & 0.033 & 42.8 & 1.2 & -119.0 & 3.7 \\
\hline 87937 & Barnard's star & 1.144 & 0.005 & 9.7 & 0.1 & -110.9 & 0.2 \\
\hline 103738 & HD 199995 & 1.254 & 1.014 & -3753.5 & 239.4 & 17.6 & 0.7 \\
\hline 100111 & HD 351880 & 1.434 & 4.617 & -944.7 & 428.1 & 26.1 & 0.3 \\
\hline 54035 & Lalande 21185 & 1.440 & 0.006 & 20.0 & 0.1 & -84.8 & 0.2 \\
\hline 94512 & HD 179939 & 1.444 & 1.496 & 3733.0 & 411.7 & -30.7 & 1.8 \\
\hline 26335 & GL 208 & 1.600 & 0.054 & -497.9 & 8.5 & 21.9 & 0.2 \\
\hline 26624 & HD 37594 & 1.637 & 0.258 & -1803.5 & 112.1 & 22.4 & 1.3 \\
\hline 27288 & GL 217.1 & 1.645 & 0.271 & -1045.6 & 162.7 & 20.0 & 3.7 \\
\hline 12351 & GJ 1049 & 1.763 & 0.494 & -609.3 & 166.2 & 26.2 & 10.0 \\
\hline 25240 & HD 35317 & 1.775 & 0.505 & -1077.7 & 73.4 & 52.6 & 1.6 \\
\hline 86963 & CD-32 13298 & 1.782 & 0.256 & 202.6 & 17.4 & -27.4 & 2.3 \\
\hline 99483 & HIP 99483 & 1.797 & 31.108 & -2889.5 & 1040.0 & 25.0 & 0.2 \\
\hline 75311 & BD-02 3986 & 1.804 & 9.702 & 3926.5 & 1120.8 & -14.3 & 0.3 \\
\hline 85605 & CCDM $17296+2439 B$ & 1.837 & 0.551 & 196.8 & 25.8 & -21.1 & 0.2 \\
\hline 47425 & GL 358 & 1.875 & 0.241 & -62.8 & 7.7 & 142.0 & 21.0 \\
\hline 92403 & Ross 154 & 1.881 & 0.080 & 151.8 & 2.4 & -11.5 & 0.8 \\
\hline 57548 & Ross 128 & 1.911 & 0.026 & 71.1 & 0.3 & -30.9 & 0.3 \\
\hline 86961 & CD-32 13297 & 1.929 & 0.371 & 189.0 & 12.8 & -28.9 & 0.9 \\
\hline 110893 & GL 860A & 1.949 & 0.042 & 88.6 & 0.6 & -33.8 & 0.2 \\
\hline 40317 & HD 68814 & 1.950 & 1.324 & -2345.0 & 264.5 & 34.2 & 0.2 \\
\hline 23641 & HD 33487 & 2.001 & 0.358 & 1040.2 & 123.9 & -39.0 & 5.0 \\
\hline 30067 & HD 43947 & 2.015 & 0.125 & -666.4 & 16.2 & 40.2 & 0.1 \\
\hline 21386 & HD 26367 & 2.019 & 0.276 & 704.4 & 40.5 & -50.7 & 1.4 \\
\hline 35550 & GL 271A & 2.044 & 1.092 & 1137.8 & 102.5 & -15.3 & 1.5 \\
\hline 101573 & HIP 101573 & 2.072 & 8.931 & -4177.0 & 1246.9 & 43.7 & 0.5 \\
\hline 20359 & GL 168 & 2.074 & 0.247 & 380.5 & 21.3 & -78.5 & 0.2 \\
\hline 16537 & Epsilon Eridani & 2.135 & 0.077 & -104.8 & 1.0 & 16.8 & 1.1 \\
\hline 86214 & GL 682 & 2.140 & 0.488 & 67.4 & 13.1 & -60.0 & 21.0 \\
\hline 38228 & HD 63433 & 2.150 & 0.146 & 1325.6 & 30.3 & -15.9 & 0.2 \\
\hline 22738 & CCDM 4535-5552A & 2.202 & 0.460 & -262.2 & 50.2 & 40.1 & 10.0 \\
\hline 13772 & GL 120.1 & 2.243 & 0.256 & -429.9 & 23.7 & 50.6 & 0.7 \\
\hline 86990 & GL 693 & 2.253 & 0.311 & 42.0 & 4.9 & -115.0 & 21.0 \\
\hline 95326 & CCDM 19236-3911B & 2.262 & 2.153 & -342.9 & 143.7 & 35.6 & 0.4 \\
\hline 68634 & HD 122676 & 2.264 & 0.339 & -305.4 & 43.8 & 83.0 & 13.9 \\
\hline 13769 & GL $120.1 \mathrm{C}$ & 2.267 & 0.186 & -503.0 & 19.8 & 49.6 & 0.7 \\
\hline 77257 & GL 598 & 2.267 & 0.043 & 165.7 & 1.5 & -66.8 & 0.3 \\
\hline 8709 & WD $0148+467$ & 2.286 & 0.272 & -237.2 & 16.5 & 64.0 & 3.0 \\
\hline 26744 & HD 37574 & 2.290 & 1.429 & 6118.0 & 1707.2 & -10.0 & 3.7 \\
\hline 32349 & Sirius & 2.299 & 0.090 & 65.7 & 4.7 & -9.4 & 1.5 \\
\hline 93506 & HD 176687 & 2.299 & 0.552 & -1205.6 & 177.3 & 22.0 & 3.7 \\
\hline 113421 & HD 217107 & 2.300 & 0.274 & 1403.8 & 153.9 & -13.5 & 1.7 \\
\hline 40501 & GJ 2066 & 2.334 & 0.314 & -133.9 & 16.6 & 62.5 & 10.0 \\
\hline 31626 & HD 260564 & 2.341 & 0.312 & -405.2 & 26.4 & 82.7 & 0.2 \\
\hline 83945 & GJ 3991 & 2.415 & 0.411 & 145.4 & 22.3 & -45.0 & 10.0 \\
\hline 5643 & GL 54.1 & 2.429 & 0.205 & -74.4 & 1.9 & 28.0 & 3.7 \\
\hline 11559 & SAO 75395 & 2.469 & 2.049 & -5409.0 & 847.2 & 20.9 & 0.8 \\
\hline 25001 & HD 34790 & 2.477 & 1.809 & 4481.0 & 356.3 & -18.7 & 0.7 \\
\hline 14576 & Algol & 2.481 & 0.930 & -6878.0 & 1068.0 & 4.0 & 0.7 \\
\hline 103039 & LP 816-60 & 2.482 & 0.114 & -270.0 & 6.6 & 15.8 & 0.6 \\
\hline 33275 & HD 50867 & 2.540 & 0.873 & 3480.0 & 177.2 & -14.4 & 0.2 \\
\hline 23708 & GJ 1075 & 2.586 & 0.639 & 366.5 & 87.3 & -29.2 & 10.0 \\
\hline
\end{tabular}


Table 4. continued.

\begin{tabular}{|c|c|c|c|c|c|c|c|}
\hline $\mathrm{HIP}^{a}$ & Name $^{b}$ & $D_{\mathrm{ca}}{ }^{c}$ & $\Delta D_{\text {ca }}{ }^{d}$ & $T_{\mathrm{ca}}{ }^{e}$ & $\Delta T_{\mathrm{ca}}^{f}$ & $v_{\mathrm{r}}^{g}$ & $\sigma_{v_{\mathrm{r}}}^{h}$ \\
\hline 1463 & GL 16 & 2.596 & 0.213 & 1017.9 & 40.3 & -15.1 & 0.4 \\
\hline 85429 & VW Oph & 2.670 & 2.573 & 542.5 & 205.3 & -90.0 & 5.0 \\
\hline 97649 & GL 768 & 2.702 & 0.059 & 139.5 & 1.8 & -26.1 & 0.7 \\
\hline 107528 & HD 207164 & 2.720 & 2.989 & 9565.0 & 674.3 & -7.2 & 0.4 \\
\hline 116727 & GL 903 & 2.791 & 0.059 & 300.1 & 4.8 & -43.1 & 0.7 \\
\hline 91726 & HD 172748 & 2.801 & 0.381 & 1248.8 & 72.1 & -44.8 & 1.8 \\
\hline 6379 & GL 56.5 & 2.825 & 0.210 & 703.9 & 49.0 & -22.7 & 1.8 \\
\hline 82977 & HD 152912 & 2.860 & 4.605 & -2726.0 & 585.5 & 50.0 & 1.8 \\
\hline 117473 & GL 908 & 2.886 & 0.045 & 62.9 & 0.3 & -71.2 & 0.1 \\
\hline 116250 & HD 221420 & 2.917 & 0.328 & -1182.4 & 121.6 & 26.0 & 3.0 \\
\hline 30920 & Ross 614 & 2.929 & 0.049 & -110.9 & 0.4 & 17.9 & 0.2 \\
\hline 39986 & HD 67852 & 2.932 & 3.158 & -4366.0 & 1073.9 & 26.4 & 7.4 \\
\hline 35136 & GJ 1095 & 2.969 & 0.068 & -189.7 & 2.1 & 84.2 & 0.2 \\
\hline 37766 & Ross 882 & 3.053 & 0.084 & -160.4 & 1.4 & 26.6 & 0.2 \\
\hline 81935 & HD 150689 & 3.145 & 0.097 & 701.7 & 10.7 & -19.1 & 0.2 \\
\hline 20917 & GL 169 & 3.189 & 0.075 & 294.1 & 2.9 & -35.2 & 0.1 \\
\hline 36795 & GL 279 & 3.196 & 0.111 & -411.7 & 7.0 & 60.1 & 0.3 \\
\hline 80824 & GL 628 & 3.208 & 0.038 & 86.0 & 0.2 & -21.0 & 0.2 \\
\hline 86162 & GL 687 & 3.213 & 0.355 & 78.8 & 1.6 & -27.9 & 6.6 \\
\hline 77910 & HD 142500 & 3.247 & 1.164 & 2845.0 & 411.9 & -25.1 & 3.7 \\
\hline 29271 & GL 231 & 3.249 & 0.067 & -255.2 & 4.4 & 34.9 & 0.7 \\
\hline 80543 & HD 148317 & 3.268 & 0.877 & 2098.0 & 228.3 & -37.0 & 3.7 \\
\hline 8102 & GL 71 & 3.271 & 0.016 & 42.6 & 0.5 & -16.4 & 0.3 \\
\hline 27075 & HD 38382 & 3.271 & 0.251 & -634.7 & 43.2 & 38.7 & 2.8 \\
\hline 1242 & GL 1005 & 3.289 & 1.148 & 105.8 & 22.6 & -29.0 & 21.0 \\
\hline 3829 & Van Maanen's star & 3.327 & 0.135 & -34.3 & 0.2 & 54.0 & 3.0 \\
\hline 91438 & GL 722 & 3.384 & 0.206 & -306.6 & 16.6 & 38.6 & 2.5 \\
\hline 23913 & HD 233081 & 3.412 & 0.663 & 1842.5 & 123.3 & -27.0 & 0.3 \\
\hline 37279 & GL $280 A$ & 3.438 & 0.035 & 29.6 & 6.9 & -3.9 & 1.0 \\
\hline 1475 & GL 15A & 3.467 & 0.015 & -16.2 & 0.3 & 12.0 & 0.2 \\
\hline 85667 & GL 678 & 3.503 & 0.158 & 200.9 & 4.3 & -76.4 & 0.5 \\
\hline 91772 & GL 725B & 3.515 & 0.061 & -0.4 & 0.2 & 0.2 & 0.1 \\
\hline 33909 & HD 53253 & 3.527 & 1.261 & -3907.0 & 298.5 & 31.1 & 1.5 \\
\hline 90112 & HD 168769 & 3.540 & 1.004 & -1888.0 & 148.4 & 26.0 & 0.3 \\
\hline 39757 & HD 67523 & 3.563 & 0.103 & -394.0 & 7.4 & 46.1 & 0.7 \\
\hline 91768 & GL $725 \mathrm{~A}$ & 3.568 & 0.032 & -0.4 & 0.2 & 0.2 & 0.1 \\
\hline 7751 & GL 66 & 3.569 & 0.118 & -283.5 & 6.0 & 22.7 & 0.7 \\
\hline 36208 & Luyten's star & 3.666 & 0.021 & -13.9 & 0.1 & 18.2 & 0.1 \\
\hline 21158 & HD 28676 & 3.681 & 1.187 & -5597.0 & 231.9 & 6.8 & 0.2 \\
\hline 6003 & HD 7735 & 3.692 & 1.599 & -2219.0 & 749.6 & 31.0 & 15.5 \\
\hline 105090 & GL 825 & 3.696 & 0.025 & -19.6 & 0.5 & 24.2 & 0.9 \\
\hline 99701 & GL 784 & 3.727 & 0.070 & 124.7 & 0.8 & -31.1 & 0.7 \\
\hline 11048 & GL 96 & 3.756 & 0.113 & 279.9 & 4.0 & -37.5 & 0.3 \\
\hline 98698 & GL 775 & 3.756 & 0.229 & 372.5 & 19.3 & -31.6 & 2.0 \\
\hline 25578 & GL 203 & 3.780 & 0.507 & -103.0 & 9.6 & 66.9 & 10.0 \\
\hline 33226 & GL 251 & 3.813 & 0.062 & -123.9 & 0.3 & 22.7 & 0.2 \\
\hline 49908 & GL 380 & 3.856 & 0.021 & 68.7 & 0.1 & -25.9 & 0.1 \\
\hline 68184 & HD 122064 & 3.868 & 0.224 & 333.2 & 16.1 & -25.3 & 1.8 \\
\hline 33277 & GL 252 & 3.869 & 0.262 & 1028.6 & 58.1 & -15.6 & 1.0 \\
\hline 117748 & $\mathrm{BD}+374901 \mathrm{C}$ & 3.940 & 6.850 & -4367.0 & 1214.3 & 7.4 & 0.7 \\
\hline 30422 & HD 44770 & 3.964 & 2.432 & -1421.6 & 305.1 & 16.3 & 1.8 \\
\hline 99859 & HD 192869 & 3.979 & 1.650 & 3889.5 & 537.0 & -28.0 & 3.7 \\
\hline 101027 & GL 791.1A & 4.021 & 0.709 & -1578.0 & 260.1 & 18.4 & 3.7 \\
\hline 87777 & HD 163547 & 4.056 & 1.373 & 3344.0 & 314.0 & -43.6 & 1.8 \\
\hline 34603 & GL 268 & 4.066 & 0.137 & -97.0 & 0.5 & 37.9 & 0.5 \\
\hline 24502 & $\mathrm{HD} 33959 \mathrm{C}$ & 4.092 & 5.367 & 1828.8 & 739.3 & -13.1 & 3.0 \\
\hline 45333 & GL 337.1 & 4.117 & 0.183 & 1285.2 & 43.2 & -14.2 & 0.5 \\
\hline
\end{tabular}


Table 4. continued.

\begin{tabular}{|c|c|c|c|c|c|c|c|}
\hline $\mathrm{HIP}^{a}$ & Name $^{b}$ & $D_{\text {ca }}^{c}$ & $\Delta D_{\mathrm{ca}}{ }^{d}$ & $T_{\mathrm{ca}}{ }^{e}$ & $\Delta T_{\mathrm{ca}}^{f}$ & $v_{\mathrm{r}}^{g}$ & $\sigma_{v_{\mathrm{r}}}^{h}$ \\
\hline 75686 & GL 585.1 & 4.132 & 0.358 & 589.1 & 25.3 & -41.4 & 0.7 \\
\hline 85523 & GL 674 & 4.134 & 0.334 & 73.7 & 16.1 & -10.2 & 4.5 \\
\hline 89959 & HD 168956 & 4.134 & 1.154 & 2831.0 & 404.8 & -25.3 & 3.7 \\
\hline 80337 & GL 620.1A & 4.153 & 0.100 & -867.6 & 9.4 & 13.0 & 0.1 \\
\hline 11964 & GL 103 & 4.180 & 0.088 & -233.2 & 2.6 & 41.9 & 0.5 \\
\hline 109555 & GL 851 & 4.205 & 0.145 & 188.3 & 2.6 & -51.4 & 0.3 \\
\hline 90595 & HD 170296 & 4.256 & 1.194 & 2128.5 & 238.9 & -41.0 & 3.7 \\
\hline 80300 & GL 620.1B & 4.259 & 0.270 & -857.6 & 21.8 & 13.0 & 0.1 \\
\hline 103659 & HD 199881 & 4.355 & 2.343 & 4874.0 & 392.3 & -15.8 & 0.6 \\
\hline 27913 & GL 222 & 4.380 & 0.075 & 471.8 & 2.9 & -13.4 & 0.1 \\
\hline 79667 & HD 146214 & 4.394 & 2.644 & 4807.0 & 636.2 & -18.9 & 2.1 \\
\hline 52341 & GJ 1136A & 4.399 & 1.131 & -473.6 & 106.1 & 31.3 & 10.0 \\
\hline 94761 & GL 752A & 4.420 & 0.055 & -70.4 & 0.2 & 35.4 & 0.4 \\
\hline 87345 & HD 162102 & 4.430 & 2.444 & 3610.5 & 589.8 & -17.5 & 1.8 \\
\hline 86400 & GL 688 & 4.461 & 0.167 & -381.4 & 9.2 & 22.7 & 0.8 \\
\hline 36186 & HD 58954 & 4.467 & 1.188 & 2869.5 & 270.5 & -29.2 & 1.8 \\
\hline 90790 & GL 716 & 4.484 & 0.119 & 274.7 & 4.3 & -41.6 & 0.7 \\
\hline 23452 & HD 32450 & 4.488 & 0.143 & 351.3 & 4.8 & -17.1 & 0.4 \\
\hline 7981 & GL 68 & 4.572 & 0.080 & 134.6 & 0.9 & -33.9 & 0.7 \\
\hline 86057 & GL 680 & 4.573 & 0.771 & 183.9 & 24.0 & -40.6 & 10.0 \\
\hline 41820 & HD 71974 & 4.639 & 0.474 & 1692.8 & 63.6 & -16.1 & 0.2 \\
\hline 113020 & GL 876 & 4.690 & 0.046 & 12.1 & 0.7 & -1.8 & 0.1 \\
\hline 27693 & HD 39655 & 4.694 & 1.526 & -3392.0 & 351.6 & 29.3 & 2.5 \\
\hline 88601 & GL 702 & 4.698 & 0.117 & 75.2 & 7.6 & -9.7 & 1.5 \\
\hline 22449 & GL 178 & 4.701 & 0.193 & -211.3 & 4.2 & 24.4 & 1.5 \\
\hline 88574 & GL 701 & 4.720 & 0.101 & -150.6 & 0.9 & 32.1 & 0.6 \\
\hline 42049 & HD 72617 & 4.760 & 0.756 & -1063.6 & 134.4 & 53.0 & 7.1 \\
\hline 86374 & HD 160295 & 4.788 & 1.657 & 2912.0 & 294.6 & -41.9 & 0.9 \\
\hline 67529 & HD 120702 & 4.820 & 0.953 & 2111.5 & 162.2 & -44.0 & 1.8 \\
\hline 47878 & HD 84566 & 4.827 & 4.300 & -6801.0 & 950.2 & 21.9 & 0.5 \\
\hline 106440 & GL 832 & 4.828 & 0.201 & -51.5 & 30.5 & 4.1 & 3.0 \\
\hline 82003 & GL 638 & 4.834 & 0.073 & 230.1 & 1.0 & -31.4 & 0.1 \\
\hline 89937 & GL 713 & 4.838 & 0.033 & -155.5 & 0.2 & 32.4 & 0.1 \\
\hline 114059 & HD 218200 & 4.856 & 2.026 & -3975.0 & 574.9 & 18.0 & 2.7 \\
\hline 43670 & HD 75935 & 4.857 & 0.931 & 2060.5 & 107.6 & -18.9 & 0.3 \\
\hline 110294 & HD 239927 & 4.862 & 0.899 & 1569.8 & 161.1 & -35.5 & 3.0 \\
\hline 80459 & GL 625 & 4.896 & 0.070 & 220.6 & 0.8 & -13.0 & 0.3 \\
\hline 44333 & HD 77173 & 4.917 & 5.079 & 4909.0 & 887.0 & -31.0 & 2.9 \\
\hline 39780 & HD 67228 & 4.924 & 0.239 & 598.8 & 16.9 & -36.4 & 0.8 \\
\hline 92871 & GL 735 & 4.926 & 0.294 & 688.1 & 25.9 & -13.5 & 0.7 \\
\hline 53985 & GL 410 & 4.973 & 0.230 & 529.6 & 16.1 & -17.6 & 0.8 \\
\hline 82817 & GL 644 & 4.982 & 0.179 & -73.6 & 3.3 & 18.8 & 1.8 \\
\hline
\end{tabular}

${ }^{a}$ Hipparcos Catalogue number. ${ }^{b}$ Alternative identification. ${ }^{c}$ Distance at closest approach in pc. ${ }^{d}$ Uncertainty in the distance at closest approach in pc; see text for details of its calculation. ${ }^{e}$ Time of closest approach in $10^{3}$ yr. Positive times denote close approaches in the future; negative times in the past. ${ }^{f}$ Uncertainty in the time of closest approach in $10^{3}$ yr; see text for details of its calculation. ${ }^{g}$ Radial velocity in $\mathrm{km} \mathrm{s}^{-1} .{ }^{h}$ Uncertainty in radial velocity in $\mathrm{km} \mathrm{s}^{-1}$.

apex at the time of the closest approach and the star's position on the plane of the sky at that time in Fig. 7. The angular bins considered are each $20^{\circ}$ in width. We find that the number of encounters within $90^{\circ}$ of the solar apex direction, 77 , is practically the same as within $90^{\circ}$ of the antapex, which is 79 . The distribution can be fitted reasonably well by a sine curve, which is what would be expected for a random distribution, except for two angular intervals. These intervals are centered, respectively, at $90^{\circ}$, which shows an excess of encounters with respect to a sinusoidal distribution, and $170^{\circ}$ (the solar antapex), which shows a deficit.

In order to assess how significant is the departure from randomness, we divide the sky into eight equal-area sections and estimate if the number of encounters in each section agrees with a random distribution. The sections are symmetric with respect to the apex-antapex direction, and correspond to the following angle intervals counted 


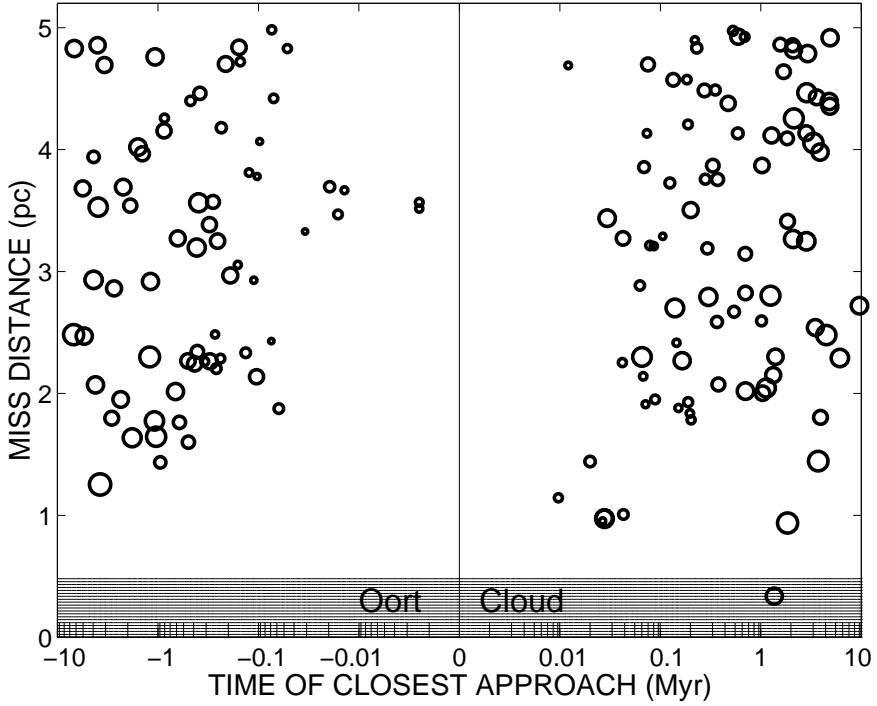

Fig. 5. Miss distance (pc) versus time (Myr) of predicted stellar approaches within $5 \mathrm{pc}$. The outer radius of the Oort cloud is approximately $10^{5} \mathrm{AU}$. The circle in the shaded area denotes the predicted future passage of GL 710 through the outer Oort cloud. The size of each circle is proportional to the star's visual brightness at closest approach (stars with bigger circles are brighter). These visual magnitudes range between -4 and 12 .

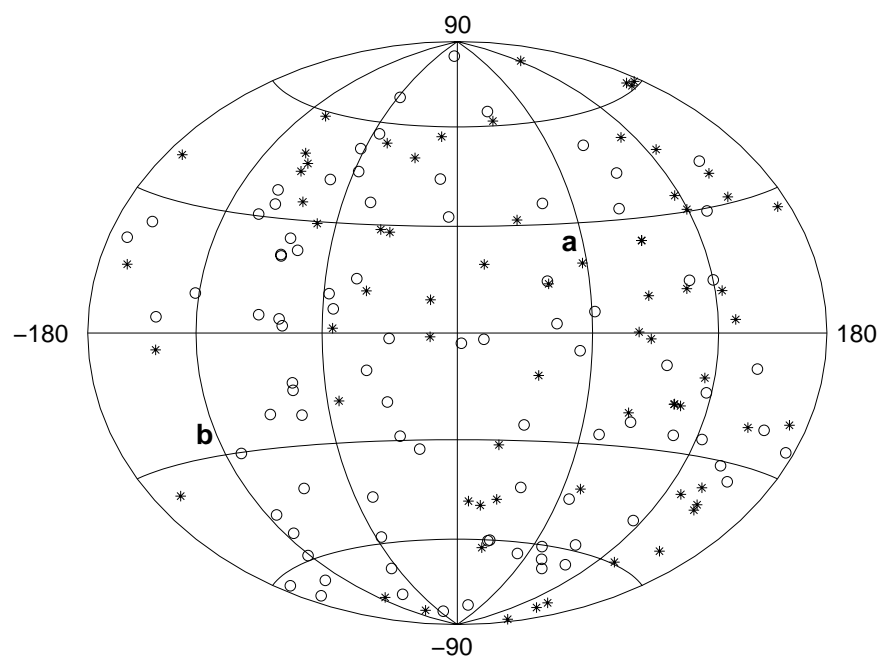

Fig. 6. Distribution of closest approach points on the plane of the sky as a function of the galactic longitude $-180^{\circ} \leq$ $l \leq 180^{\circ}$ and latitude $-90^{\circ} \leq b \leq 90^{\circ}$. The symbol "a" denotes the solar apex and "b" the solar antapex directions. Encounters in the past are denoted by an asterisk, whereas encounters in the future are denoted by a circle.

from the solar apex direction: $\left(0^{\circ}, 41.4^{\circ}\right),\left(41.4^{\circ}, 60^{\circ}\right)$, $\left(60^{\circ}, 75.5^{\circ}\right),\left(75.5^{\circ}, 90^{\circ}\right),\left(90^{\circ}, 104.5^{\circ}\right),\left(104.5^{\circ}, 120^{\circ}\right)$, $\left(120^{\circ}, 138.6^{\circ}\right)$, and $\left(138.6^{\circ}, 180^{\circ}\right)$. If $N=156$ is the total number of encounters within 5 pc and $s=8$ is the number of sections, then the mean number of encounters in each section is the ratio $N / s$, or $\lambda=19.5$. Assuming a Poisson distribution, the standard deviation is $\sigma=\lambda^{1 / 2}=$ 4.4. The departure from randomness is not significant if the difference between the number of encounters in each

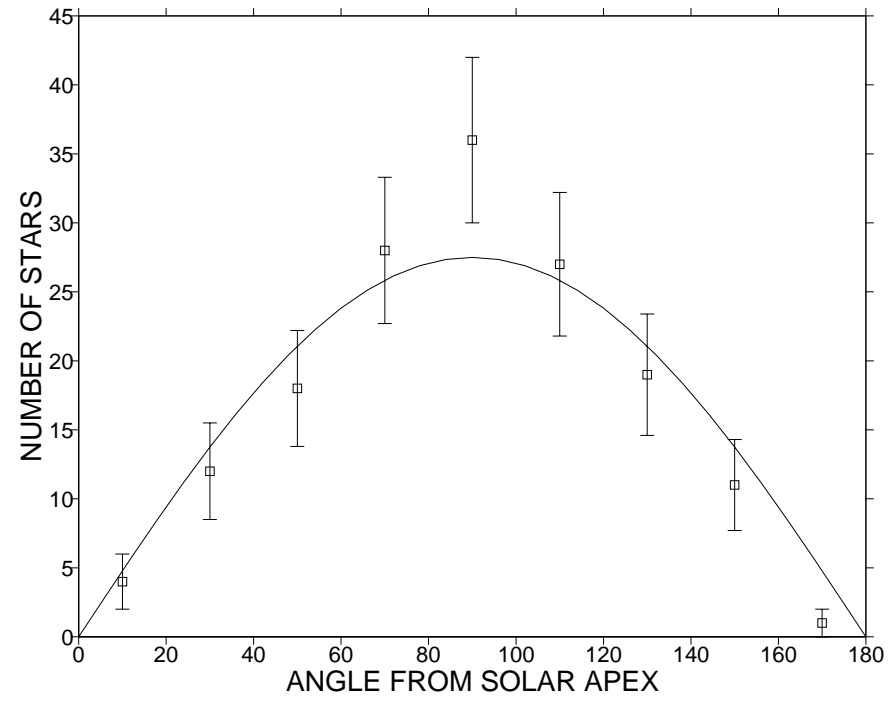

Fig. 7. Number of encounters within 5 pc as a function of the angle (in degrees) between the solar apex and the star position on the plane of the sky at the time of closest approach. The values are taken in bins of $\pm 10^{\circ}$. The error bars plotted are $\pm \sqrt{n}$, where $n$ is the value of the data point. The continuous line represents a sinusoidal distribution.

Table 5. Number of encounters in each section and deviation from the mean.

\begin{tabular}{ccc}
\hline Angular interval & Number of encounters & $\mid$ Value $-\left.\lambda\right|^{a}$ \\
\hline$\left(0^{\circ}, 41.4^{\circ}\right)$ & 17 & $0.6 \sigma$ \\
$\left(41.4^{\circ}, 60^{\circ}\right)$ & 17 & $0.6 \sigma$ \\
$\left(60^{\circ}, 75.5^{\circ}\right)$ & 22 & $0.6 \sigma$ \\
$\left(75.5^{\circ}, 90^{\circ}\right)$ & 21 & $0.3 \sigma$ \\
$\left(90^{\circ}, 104.5^{\circ}\right)$ & 26 & $1.5 \sigma$ \\
$\left(104.5^{\circ}, 120^{\circ}\right)$ & 22 & $0.6 \sigma$ \\
$\left(120^{\circ}, 138.6^{\circ}\right)$ & 19 & $0.1 \sigma$ \\
$\left(138.6^{\circ}, 180^{\circ}\right)$ & 12 & $1.7 \sigma$ \\
\hline
\end{tabular}

${ }^{a}$ Difference between the value in Col. 2 and $\lambda=19.5$ in units of sigma.

equal-area section and the expected number of encounters $\lambda$ is small. We list the number of encounters in each section as well as their difference with respect to $\lambda$ in Table 5 . We find that the difference is larger than one sigma in two sections. For the interval $\left(90^{\circ}, 104.5^{\circ}\right)$ the number of encounters is $1.5 \sigma$ above the expected mean value, and for $\left(138.6^{\circ}, 180^{\circ}\right)$ the number of encounters is $1.7 \sigma$ below the expected mean value.

Although the values in these two sections might give some evidence of non-randomness, the departure from a random distribution is not significant. Since $\lambda=19.5$ is large, the Poisson distribution can be well approximatted by a Gaussian distribution with $\sigma=\lambda^{1 / 2}=4.4$. For a Gaussian distribution, $68.27 \%$ of the cases are expected to lie within $1-\sigma, 86.64 \%$ within $1.5-\sigma$, and $95.45 \%$ within $2-\sigma$. We find that $75 \%$ of the cases lie within $1-\sigma$, $87.5 \%$ within $1.5-\sigma$, and $100 \%$ within $2-\sigma$ (see Table 5 ), which shows that the distribution is essentially Gaussian. 

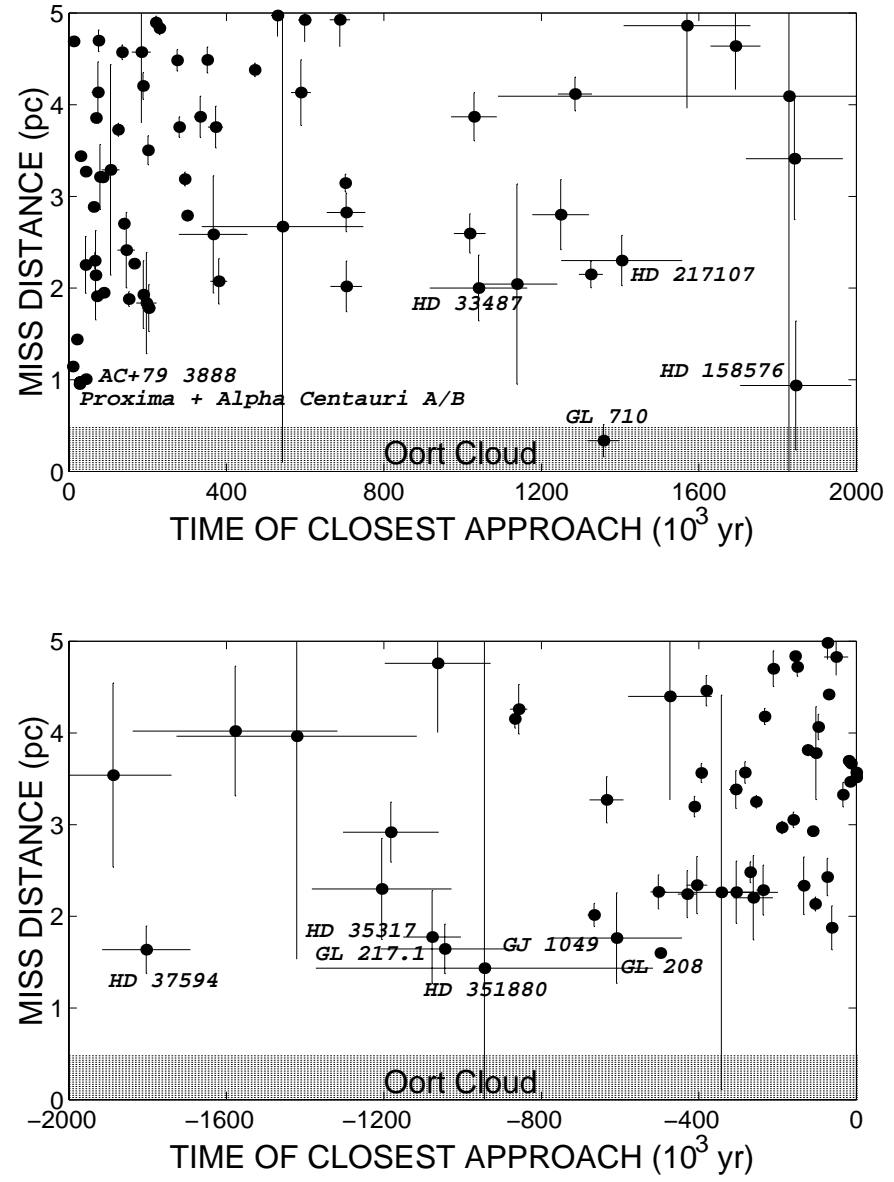

Fig. 8. Predicted stellar encounters with the Sun within 5 pc, $2 \mathrm{Myr}$ in the future (top) and $2 \mathrm{Myr}$ in the past (bottom). The error bars are $\pm \Delta D_{\text {ca }}$ and $\pm \Delta T_{\text {ca }}$, as given by Eq. (27) and (28). Names of several of the stars are noted.

The Sun's velocity relative to the LSR is about half the typical value of a star of its type, and smaller than typical velocities of most of the stellar types encountered, so in general the effect of the solar motion direction on the distribution of encounters on the sky is expected not to be significant. Nevertheless, all the values around $90^{\circ}$ of the solar direction in Table 5 are above the mean number of encounters, whereas all the values around the solar apex or antapex direction are below the mean, which might indicate some effect of the solar motion direction. However, as seen above, this possibility is not statistically significant.

Figure 8 shows plots of the past and future stellar passages, respectively, within a time interval of $\pm 2 \mathrm{Myr}$ from the present time. The shaded area at the bottom of each plot represents the Oort cloud. Error bars given by $\pm \Delta D_{\text {ca }}$ and $\pm \Delta T_{\text {ca }}$ are shown. The size of some error bars is smaller than the dot size. Most of the encounters are at times close to the present time. As the time of encounter increases the number of encounters decreases. This is further evidence of the observational incompleteness mentioned above. For some stars the uncertainty in the predicted passage is very large, so the predicted encounter parameters should be considered with caution.
Table 6. Time of closest approach $T_{\text {ca }}$ (in $10^{3}$ yr) and miss distance (in pc) of GL 710 determined using different models.

\begin{tabular}{lll}
\hline Local potential model & $1357.6 \pm 40.9$ & $0.337 \pm 0.177$ \\
Global potential model & $1357.6 \pm 40.9$ & $0.337 \pm 0.177$ \\
Spiral perturbation model & $1358.0 \pm 40.9$ & $0.334 \pm 0.177$ \\
\hline
\end{tabular}

We find that the candidate star with the closest approach to the solar system is GL 710 (HIP 89825), a KV star with a predicted minimum distance of $0.337 \pm 0.177 \mathrm{pc}$ in $1.36 \pm 0.04 \mathrm{Myr}$. This confirms our result in Paper I that GL 710 will have a passage through the outer Oort cloud, where we concluded that the perturbation caused by this passage is not strong enough to disrupt the cometary cloud. A close passage by this star was first predicted by Vyssotsky (1946) based on its low proper motion. This star is the only one of our candidate stars with a miss distance less than $10^{5} \mathrm{AU}(\sim 0.5 \mathrm{pc})$. In Table 6 we compare the results for GL 710 with those obtained using the other galactic potential models described above, assuming the same values of the galactic parameters. These results show that the predicted encounter with GL 710 is essentially independent of the model adopted. For the spiral perturbation model we use the two-armed configuration with two different values of the pattern speed of rotation of the spiral arms, $\Omega_{\mathrm{p}}=13.5$ and $20.0 \mathrm{~km} \mathrm{~s}^{-1} \mathrm{kpc}^{-1}$. For both values of $\Omega_{\mathrm{p}}$ we obtain the same encounter parameters. We find an excellent agreement between the local and global potential models for the encounter with GL 710 . For the calculation of the encounter parameters we assume that GL 710 is a single star. In Paper I we discussed the possible binary nature of GL 710, suspected because of the larger values of the radial velocity measurements made more than 50 years ago. We concluded that the difference in radial velocity may be due to a systematic error in the zero point of the measurements made in the 1940's. However, we also cautioned that additional monitoring of the radial velocity and the astrometric positions over the coming years is desirable to settle this conclusion.

The second closest passage is that of HD 158576 (HIP 85661), an F0 star with a predicted miss distance of $0.938 \mathrm{pc}$ in $1.85 \mathrm{Myr}$. The third closest predicted approach is for the M5.5 dwarf Proxima Centauri (HIP 70890), currently the nearest star to the Sun, for which the predicted closest passage is $0.954 \mathrm{pc}$ in $26700 \mathrm{yr}$. All of the above passages are predicted for the future. The closest passage in the past was that of the G8III star HD 199995 (HIP 103738), with a predicted miss distance of $1.254 \mathrm{pc}$ and time of closest approach of $3.8 \mathrm{Myr}$ in the past. Eleven stars in Table 4 have errors $\Delta D_{\text {ca }}$ that are larger than their predicted closest approach distances $D_{\text {ca. }}$. These are stars for which improved data would be particularly useful.

The closest passage of a star with an extrasolar planet candidate was that of Epsilon Eridani (HIP 16537). This K2 V star, recently proposed as a candidate for having a Jupiter-like companion of mass $M \sin i=0.86 M_{\mathrm{Jup}}$ 
(Hatzes et al. 2000), passed at a distance of 2.14 pc about $0.105 \mathrm{Myr}$ ago. Encounters within $5 \mathrm{pc}$ with other candidates for extrasolar planets are also predicted. HD 217107 (HIP 113421), a G7 V star with a planetary companion of mass $M \sin i=1.27 M_{\text {Jup }}$ (Fischer et al. 1999), presently located at $19.7 \mathrm{pc}$, will pass at a heliocentric distance of $2.3 \mathrm{pc}$ in $1.4 \mathrm{Myr}$ in the future. GL 876 (HIP 113020), an M4 dwarf star located at $4.702 \mathrm{pc}$ with a planetary companion of mass $M \sin i \simeq 2.0 M_{\text {Jup }}$ (Delfosse et al. 1998), will pass at a distance of $4.69 \mathrm{pc}$ in about $12100 \mathrm{yr}$.

\section{Comparison between potential models}

Once we have determined the encounter parameters (miss distance and time of closest approach) for the candidate stars with the solar system using the global potential model, we are interested in studying the limits of validity of the predictions by this potential model by comparing with the other two models considered. We particularly focus our attention on the candidate stars encountering the Sun within $5 \mathrm{pc}$ in a time interval of $\pm 10 \mathrm{Myr}$, which are listed in Table 4. The encounters predicted for these candidate stars will be used later to derive the frequency of encounters with the solar system.

\subsection{Comparison between global potential and local potential models}

We determined the stellar encounters using both global and local potential models. For the local potential model we adopted the same values of the Sun's galactocentric distance, $R_{\odot}=8.0 \mathrm{kpc}$, and the local mass density, $\rho_{\odot}=$ $0.143 M_{\odot} \mathrm{pc}^{-3}$, adopted for the global potential model.

We compare the results obtained from both models in Fig. 9. The difference $\Delta D$ between the miss distances determined by the global and the local potential models, as a function of the time of closest approach $T_{\mathrm{ca}}$, for the 156 candidate stars passing within $5 \mathrm{pc}$, is shown in Fig. 9a. For these stars the agreement between the two models is very good. The maximum difference is less than $0.2 \mathrm{pc}$, and for the overwhelming majority of stars the agreement is better than 0.03 pc. For most of the closest passages (within $2 \mathrm{pc}$ ) the agreement is practically perfect. The local potential model predicts passages within $5 \mathrm{pc}$ of the Sun for the same candidate stars as the ones predicted by the global potential model, with the sole exception of HIP 44333. The passage for this star is predicted by the local potential model to be at a distance of $5.111 \mathrm{pc}$ in $4.9 \mathrm{Myr}$, slightly greater than $5 \mathrm{pc}$, as compared to the $4.917 \mathrm{pc}$ in $4.9 \mathrm{Myr}$ predicted by the global potential model. This star exhibits the maximum difference in the estimated miss distances $(\Delta D \sim 0.2 \mathrm{pc}$ in the plot) between the two models.

Figure $9 \mathrm{~b}$ shows $\Delta D$ as a function of $T_{\text {ca }}$ for all 595 candidate stars. We see that for stellar passages within $\sim 10 \mathrm{Myr}$, the local and the global potential results are in good agreement. The differences are below $0.5 \mathrm{pc}$, except for three stars for which the differences are between $0.5 \mathrm{pc}$ and $1.13 \mathrm{pc}$; these differences represent less than $8 \%$ of the actual miss distances. However, for encounter times larger than about $\sim 10 \mathrm{Myr}$ (noted in the plot by a vertical line) the model predictions begin departing for several stars, a few of them significantly. The distribution of the 595 candidate stars as a function of $\Delta D$ is shown in Fig. 9c. We use bins of different width to better show the good agreement between the models for a large number of stars. Only $\sim 5 \%$ of the 595 stars have $\Delta D$ larger than $0.5 \mathrm{pc}$. The fact that the models show such a good agreement for the time interval of integration of $\pm 10 \mathrm{Myr}$ will be used later to study the variation of the predicted encounters by the adoption of different galactic parameters.

We discussed above the validity of the harmonic motion of the stars above and below the galactic midplane implied by the local potential model. The harmonic approximation cannot be used reliably for stars with amplitudes of vertical motion larger than $\sim 300 \mathrm{pc}$ from the midplane. However, for our candidate stars with amplitudes larger than $300 \mathrm{pc}$, they only reach these amplitudes long after they have encountered the Sun. The difference in the predicted encounter parameters between the local and the global potential models is very small, practically zero for all of them.

\subsection{Effect of a spiral potential perturbation on the stellar orbits}

We can also study the effect of the potential of the spiral arms on the stellar passages predicted by the global potential model. First we determine the encounters within the $\pm 100 \mathrm{Myr}$ time interval of integration by using the two values of the pattern speed, $\Omega_{\mathrm{p}}=13.5 \mathrm{~km} \mathrm{~s}^{-1} \mathrm{kpc}^{-1}$ and $\Omega_{\mathrm{p}}=20.0 \mathrm{~km} \mathrm{~s}^{-1} \mathrm{kpc}^{-1}$ discussed above. We find a very good agreement between the miss distances obtained for both values of $\Omega_{\mathrm{p}}$. For 582 of the 595 candidate stars the agreement in encounter distance is within 0.1 pc. For the other candidate stars the differences are between 0.1 and $3 \mathrm{pc}$ (only two stars have a difference larger than $1 \mathrm{pc}$ ) but they represent less than $4 \%$ the value of the miss distance in all but one case. The only exception is HIP 112584, with a difference of $\sim 55 \%$ in the value of the miss distance. However, the predicted closest passage for this star is larger than $23 \mathrm{pc}$ for either of the two values of $\Omega_{\mathrm{p}}$, so this disagreement does not affect the encounters within $5 \mathrm{pc}$ of the Sun. This star has the lowest heliocentric velocity in our sample, less than $1 \mathrm{~km} \mathrm{~s}^{-1}$. If we adopt a pattern speed of $\Omega_{\mathrm{p}}=20.0 \mathrm{~km} \mathrm{~s}^{-1} \mathrm{kpc}^{-1}$, we must integrate the orbit of HIP 112584 beyond the 100 Myr interval of integration to determine its encounter with the Sun. We find a much better agreement with the global potential prediction for this star when we adopt an $\Omega_{\mathrm{p}}$ of $13.5 \mathrm{~km} \mathrm{~s}^{-1} \mathrm{kpc}^{-1}$. The differences in the integrations likely result from the very low relative velocity and long integration time, allowing small differences in the potential models to grow significantly. With this sole exception of HIP 112584, we can assume that for the candidate stars 

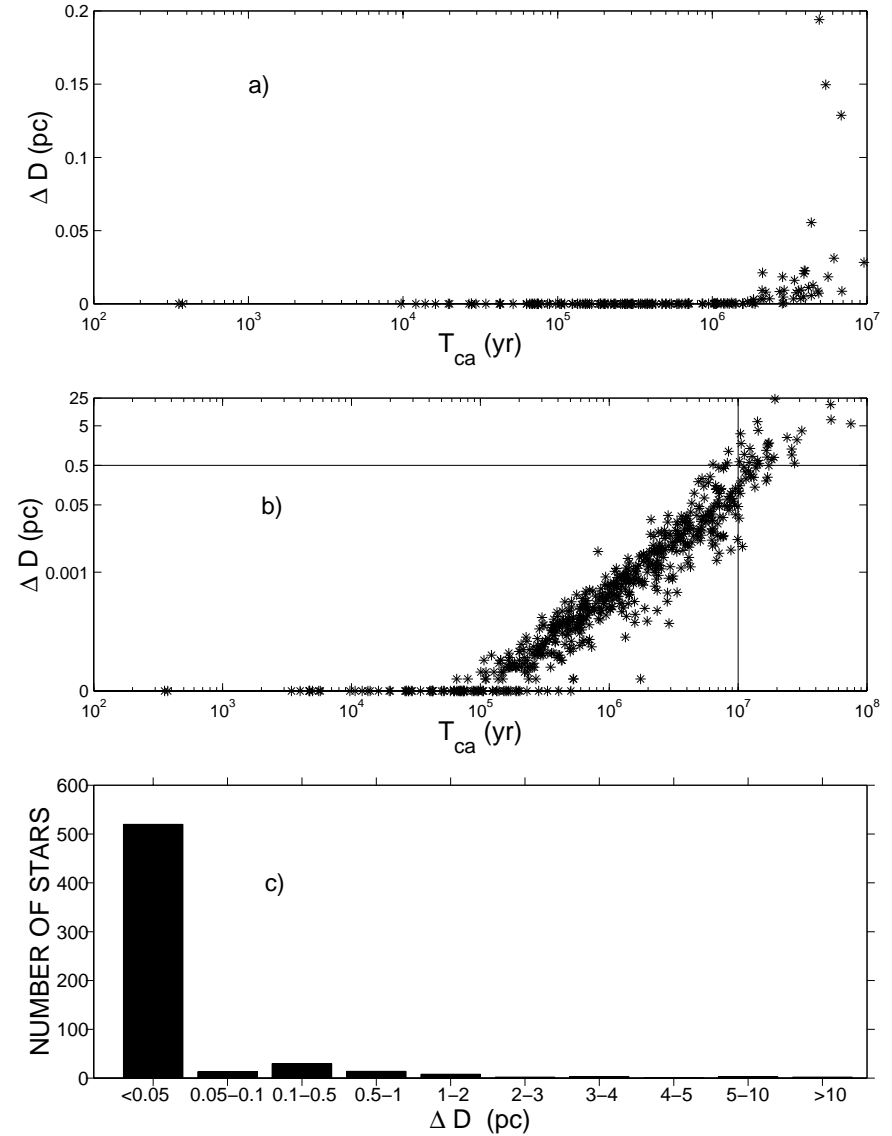

Fig. 9. a) Difference $\Delta D$ in pc between the miss distances using the global and the local potential models, as a function of time of closest approach $T_{\text {ca }}$ in yr (logarithmic axis), for the 156 stars encountering the Sun within 5 pc. b) $\Delta D$ in pc (logarithmic axis) versus $T_{\text {ca }}$ in yr (logarithmic axis) for all 595 candidate stars. Horizontal line marks $\Delta D=0.5$ pc. Vertical line marks $T_{\mathrm{ca}}=$ 10 Myr. c) Number of stars as a function of $\Delta D$ for the 595 candidate stars. Note that different bin widths are used.

the spiral perturbation model predictions do not differ significantly due to the use of one or the other values of $\Omega_{\mathrm{p}}$ considered.

Figure 10a shows the difference $\Delta D$ for the global and spiral arm models as a function of time $T_{\text {ca }}$ for the 156 candidate stars encountering the Sun within 5 pc, assuming a pattern speed $\Omega_{\mathrm{p}}=13.5 \mathrm{~km} \mathrm{~s}^{-1} \mathrm{kpc}^{-1}$. The predictions of the global potential model are not significantly affected by the spiral arms potential for most of the passages. The agreement is within $0.1 \mathrm{pc}$ for most of the passages, and the maximum difference is less than 0.4 pc. Both models predict the same candidate stars passing within $5 \mathrm{pc}$ of the Sun. The effect of the spiral arms on the encounters predicted within 5 pc can thus be neglected. The agreement is very good for encounters within about \pm 2 Myr from the present time.

Figure $10 \mathrm{~b}$ shows the difference $\Delta D$ as a function of time $T_{\text {ca }}$ for all 595 candidate stars. We see that the effect of the spiral arms is significant for passages with large encounter times. For passages within about \pm 7 Myr the
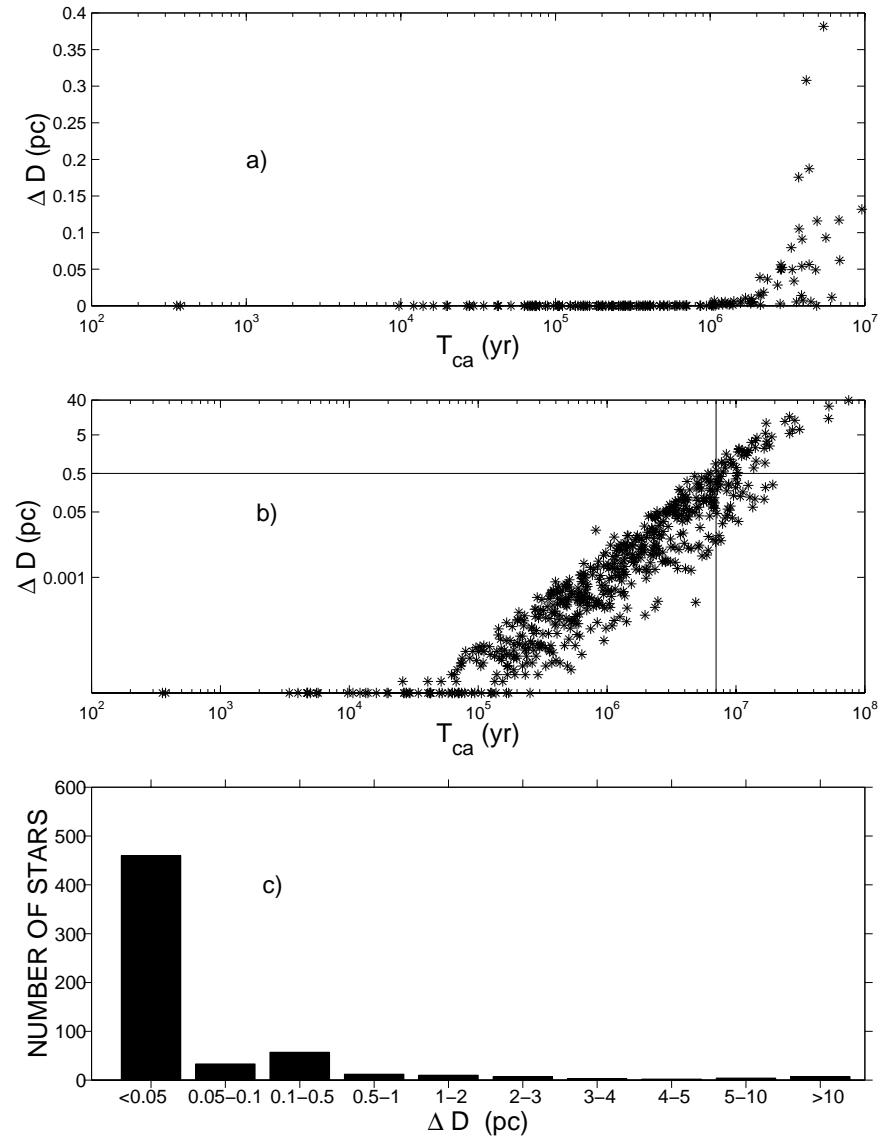

Fig. 10. a) Difference in miss distance $\Delta D$ in pc between the global potential model with and without a two-armed spiral perturbation, as a function of $T_{\text {ca }}$ in yr (logarithmic axis), for the 156 stars encountering the Sun within 5 pc. b) $\Delta D$ in pc (logarithmic axis) versus $T_{\text {ca }}$ in yr (logarithmic axis) for the 595 candidate stars. Horizontal line marks $\Delta D=0.5 \mathrm{pc}$. Vertical line marks $T_{\mathrm{ca}}=$ 7 Myr. c) Number of stars as a function of $\Delta D$ for all 595 candidate stars. Note that different bin widths are used.

difference $\Delta D$ is smaller than $0.5 \mathrm{pc}$. The vertical line in the plot denotes this time. For larger encounter times, $\Delta D$ increases roughly steadily with time for most of the passages. The distribution of the 595 candidate stars as a function of $\Delta D$ is shown in Fig. 10c. We see that, although $\Delta D$ is significant for some passages, for $\sim 92 \%$ of the 595 stars the value of $\Delta D$ is smaller than $0.5 \mathrm{pc}$.

\subsection{Dependence of results on the values of the galactic parameters}

We described above a range of plausible values for the parameters in the local potential model, and we also saw that the encounters predicted with the local potential model matched well those predicted by the global model. The simplicity in the use of the local potential model, where a range of values of the galactic parameters may be tested, is an advantage to predict the sensitivity of the stellar encounters to these parameters as compared with the global model. Thus, we use the local potential model, along with its plausible range of parameters, to study this sensitivity. 
We tested different values of the Sun's height above the midplane, within the range $z_{\odot}=0-20 \mathrm{pc}$, and we found no significant difference in the values of the encounter parameters with respect to the ones determined using the value of $z_{\odot}=10 \mathrm{pc}$ adopted for our integrations. For the extreme values of $z_{\odot}$ above, the average difference between the miss distances is less than $0.02 \%$. Therefore, within this range the dependence of the encounters on the value of $z_{\odot}$ can be neglected.

For the distance to the galactic center we considered $R_{\odot}=7.5-8.5 \mathrm{kpc}$. We found no significant change in miss distance due to the use of values within this range. The difference is well below $0.01 \mathrm{pc}$ for almost all passages. The maximum difference is 0.155 pc for HIP 79333 , which is only $3 \%$ of the miss distance determined for this star. Therefore, we conclude that the effect of using values of $R_{\odot}$ different than $8.0 \mathrm{kpc}$ within the range considered above can be neglected.

We adopted a range of $\rho_{\odot} \simeq 0.076-0.15 M_{\odot} \mathrm{pc}^{-3}$ for the local mass density. As test values for this study we consider the value $\rho_{\odot}=0.143 M_{\odot} \mathrm{pc}^{-3}$ adopted for our integrations, which represents a high value of $\rho_{\odot}$, and the value $\rho_{\odot}=0.076 M_{\odot} \mathrm{pc}^{-3}$, which is the lower limit of the range considered. The difference $\Delta D$ between the miss distances predicted by the two values of $\rho_{\odot}$, as a function of the time of closest approach, is plotted in Fig. 11. The difference $\Delta D$ for the candidate stars with passages within $5 \mathrm{pc}$ of the Sun is shown in Fig. 11a. The plot shows a good agreement, $\Delta D \leq 0.05 \mathrm{pc}$, for most of the encounters. For most of the closest passages (within $2 \mathrm{pc}$ ) the agreement is very good. Only eight stars have $0.05 \mathrm{pc}<$ $\Delta D \leq 2.2 \mathrm{pc}$. The lower value of $\rho_{\odot}$ results in the addition of two passages to the list of encounters within $5 \mathrm{pc}$, whereas two other passages are removed from this list. None of the passages added or removed are within $\sim 4 \mathrm{pc}$ or within $\pm 3 \mathrm{Myr}$.

Figure $11 \mathrm{~b}$ shows $\Delta D$ as a function of the time of closest approach for all 595 candidate stars. We see that the difference in the predictions for the two values of $\rho_{\odot}$ is significant for several encounters. The vertical line denotes a time of $\pm 10 \mathrm{Myr}$, within which the local and the global potential model are in good agreement (see Sect. 6.1). The number of stars as a function of $\Delta D$ for encounters within $\pm 10 \mathrm{Myr}$ is plotted in Fig. 11c. Within \pm 10 Myr from the present time the effect of varying the local mass density $\rho_{\odot}$ on the stellar trajectories is a change of less than $0.05 \mathrm{pc}$ in the miss distance for $\sim 73 \%$ of the passages. This change is larger than $0.5 \mathrm{pc}$ for only $\sim 8 \%$ of the stars passing within \pm 10 Myr.

\subsection{Galactic parameters uncertainty versus astrometric errors comparison}

We are interested in estimating the main source of uncertainty affecting our results. As sources of uncertainty we consider those arising from the values of the galactic parameters adopted for the potential model, and the
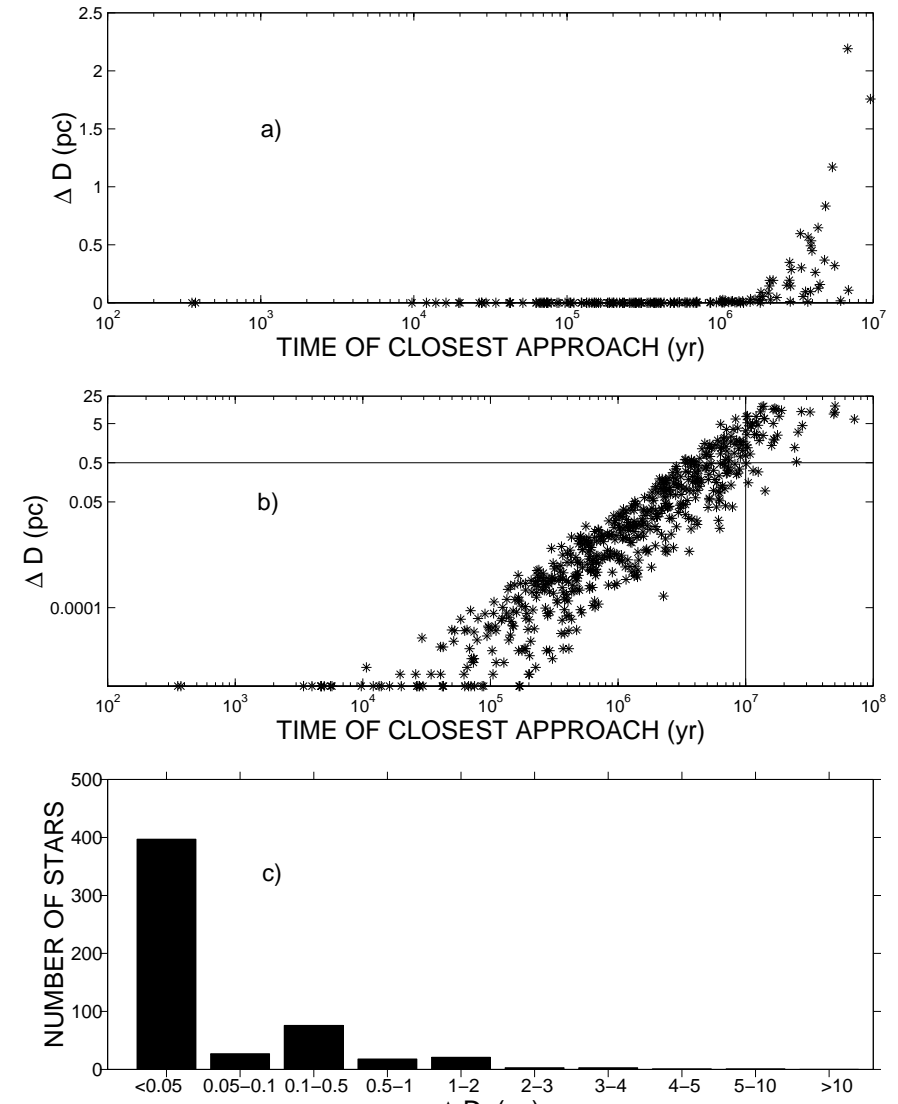

$\Delta \mathrm{D}(\mathrm{pc})$

Fig. 11. a) Difference $\Delta D$ in pc between encounters predicted using $\rho_{\odot}=0.076 M_{\odot} \mathrm{pc}^{-3}$ and those using $\rho_{\odot}=$ $0.143 M_{\odot} \mathrm{pc}^{-3}$, for the local potential model, as a function of the time of closest approach $T$ in yr (logarithmic axis), for the 156 candidate stars encountering the Sun within 5 pc. b) $\Delta D$ in pc (logarithmic axis) versus $T$ in yr (logarithmic axis) for all 595 candidate stars. Horizontal line marks $\Delta D=0.5$ pc. Vertical line marks $T=10$ Myr. c) Number of stars as a function of $\Delta D$ for encounters within $\pm 10 \mathrm{Myr}$. Note that different bin widths are used.

observational errors on the astrometric parameters of the candidate stars.

We found that the largest changes in the predicted encounter parameters due to the galactic parameters used are for the adoption of a different value of the local mass density $\rho_{\odot}$, and for the contribution of the potential of the spiral arms. These two effects limit the accuracy of the stellar trajectories calculated with the global potential model. For the astrometric errors we consider the errors associated with the measured values of parallax, proper motion in right ascension, proper motion in declination and radial velocity.

To estimate whether our predictions are limited more by the potential model adopted or by the observational errors of the candidate stars, we consider $\Delta D_{\text {obs }}$ and $\Delta T_{\text {obs }}$, the uncertainties in the prediction of the miss distance and the time of closest approach, respectively, due to the astrometric errors, and $\Delta D_{\bmod }$ and $\Delta T_{\bmod }$, the uncertainties in the predictions of the miss distance and the time of closest 
approach, respectively, due to the potential model (galactic parameters) adopted. The values of $\Delta D_{\text {obs }}$ and $\Delta T_{\text {obs }}$ are given by Eqs. (27) and (28). The values of $\Delta D_{\bmod }$ and $\Delta T_{\bmod }$ are calculated as

$\Delta D_{\text {mod }}=\left|D_{\text {ca }}-D_{\text {ca }}^{\star}\right|$

$\Delta T_{\text {mod }}=\left|T_{\mathrm{ca}}-T_{\mathrm{ca}}^{\star}\right|$

where $D_{\text {ca }}$ and $T_{\text {ca }}$ are the miss distance and the time of closest approach determined by the global potential model, respectively, and $D_{\text {ca }}^{\star}$ and $T_{\text {ca }}^{\star}$ are the miss distance and time of closest approach determined for a smaller local mass density $\rho_{\odot}$ or for the contribution of the potential of the spiral arms. The predicted encounters are constrained by the observations when $\Delta D_{\text {obs }}>\Delta D_{\bmod }$ or when $\Delta T_{\text {obs }}>\Delta T_{\text {mod }}$, whereas they are constrained by the potential model when $\Delta D_{\text {obs }}<\Delta D_{\text {mod }}$ or when $\Delta T_{\text {obs }}<\Delta T_{\bmod }$. We study the effect of each of the parameters separately.

We compare the effect of a value of $\rho_{\odot}=$ $0.076 M_{\odot} \mathrm{pc}^{-3}$ on the predicted encounters with the effect of the astrometric errors. The percentage of stars with $\Delta D_{\text {obs }}>\Delta D_{\text {mod }}$ is $\sim 99 \%$ of the total number of stars. The percentage is about the same for $\Delta T_{\text {obs }}>\Delta T_{\bmod }$, $\sim 97 \%$. We also compare the effect of the spiral arms of the Galaxy on the predicted encounters with the effect of the astrometric errors. We find that $\sim 99 \%$ of the stars have $\Delta D_{\text {obs }}>\Delta D_{\text {mod }}$. The percentage is the same for $\Delta T_{\text {obs }}>\Delta T_{\text {mod }}$. This shows that the errors in the astrometric parameters are the main effect limiting the accuracy of the predicted passages.

\section{Frequency of encounters and completeness of the study}

We wish to find the frequency of encounters of the Sun with star systems, where a star system is defined to contain either one or more stars. Unless we deal with a penetrating passage of a wide binary or multiple system through the Oort cloud, in which case the geometry of the system would need to be taken into account, each passage is counted as a single encounter for dynamical purposes.

The frequency of stellar passages within any distance, $D$, of the Sun can be estimated by

$f=\frac{N}{t}=\pi D^{2} v n_{*}$

where $v$ is the velocity of encounter of the Sun with the stellar systems and $n_{*}$ is the local density of stellar systems (Jeans 1928; see also Weissman 1980; Hills 1981; Stern \& Shull 1988). The velocity $v$ not only depends on the Sun's peculiar velocity $v_{\odot}$, but also on the velocity dispersions of the stars encountered, $v_{*}$, since the stars are not at rest in space. We combine $v_{\odot}$ and $v_{*}$ in quadrature to define the velocity of encounter, $v=\sqrt{v_{\odot}^{2}+v_{*}^{2}}$ (e.g., Hut \& Tremaine 1985). We can refine this estimate by determining the Sun's encounter frequency with different stellar types, each characterized by its own stellar velocity dispersion, density and solar motion, and adding them to obtain the total frequency of encounters. The frequency of encounters can thus be rewritten as

$f=\frac{N}{t}=\pi D^{2} \sum_{i} v_{i} n_{* i}$

where index $i$ denotes the different stellar types considered, and with relative velocity $v_{i}=\sqrt{v_{\odot i}^{2}+v_{* i}^{2}}$ for each type.

For this calculation, we require the number density $n_{* i}$ for each stellar type. This number density can be obtained from the luminosity function (LF) of star systems (the systemic LF) for the $M_{V}$ interval representing each stellar type. Using data from the literature, we construct a LF as close as possible to a representative systemic LF, from which the number density can be derived.

We consider data from Gould et al. (1997) for faint stars and from Jahreiss \& Wielen (1997) for main sequence stars. The values from Jahreiss \& Wielen are based on data for the preparation of the new Catalogue of Nearby Stars, which also uses Hipparcos parallaxes for those catalogue stars observed by the Hipparcos satellite. This catalogue contains stars within $25 \mathrm{pc}$, and it is complete for $M_{V}$ brighter than about magnitude 9 out to $25 \mathrm{pc}$. The values from Gould et al. are based on star counts for the faint end of the luminosity function. The advantage of using Gould et al.'s data is that all secondaries in binary systems are essentially missed (Frogel \& Gould 1998), providing a good estimate of the systemic LF. We construct a LF based on Jahreiss \& Wielen (1997) data for the interval $-1 \leq M_{V} \leq 9$, and on Gould et al. (1997) data for the interval $10 \leq M_{V} \leq 18$. This LF is listed in Table 7 . From this LF we calculate the number density corresponding to the $M_{V}$ interval representing each stellar type.

We also consider number densities for other stellar types. For white dwarf stars, Liebert et al. (1988) found a value of $3.2 \times 10^{-3} \mathrm{pc}^{-3}$ based on data for 43 white dwarfs contained in the Luyten Half-Second Catalogue (LHS, Luyten 1979). Leggett et al. (1998) used better measurements of the same sample, and reported a value of $3.4 \times 10^{-3} \mathrm{pc}^{-3}$. Ruiz et al. (1993) found a factor of two larger for the number of stars observed in some areas of the sky with respect to the same areas in the LHS catalogue. This suggests a possible observational incompleteness in the LHS catalogue. Consequently, we take the Leggett et al. (1998) value and we assume it to represent a lower limit. We then correct for the fraction of these white dwarfs that are not the brightest components of binary systems, and we obtain a value of $\sim 3 \times 10^{-3} \mathrm{pc}^{-3}$, which we adopt as the number density of white dwarfs.

For giant stars we adopt a number density of $4.3 \times 10^{-4} \mathrm{pc}^{-3}$, which is the difference between the general LF (for the $M_{V}$ interval containing giants plus main sequence stars) and the main sequence LF reported in Jahreiss \& Wielen (1997). This value is very close to the value of $4.6 \times 10^{-4} \mathrm{pc}^{-3}$ we derive from the $\mathrm{LF}$ 
Table 7. Luminosity function $\Psi\left(M_{V}\right)$ adopted for the range $-1 \leq M_{V} \leq 18$, in units of $10^{-3} \mathrm{pc}^{-3}$.

\begin{tabular}{|c|c|c|c|c|c|c|c|c|c|c|c|c|c|c|c|c|c|}
\hline$M_{V}$ & $=$ & -1 & 0 & 1 & 2 & 3 & 4 & 5 & 6 & 7 & 8 & 9 & 10 & 11 & 12 & 13 & 14 \\
\hline$\Psi\left(M_{V}\right)$ & $=$ & 0.015 & 0.09 & 0.24 & 0.42 & 1.10 & 1.60 & 2.92 & 2.98 & 2.92 & 3.34 & 4.17 & 7.1 & 9.5 & 10.1 & 6.3 & 3.9 \\
\hline$M_{V}$ & $=$ & 15 & 16 & 17 & 18 & & & & & & & & & & & & \\
\hline$\Psi\left(M_{V}\right)$ & $=$ & 1.9 & 1.7 & 2.3 & 2.3 & & & & & & & & & & & & \\
\hline
\end{tabular}

Table 8. Contribution of each stellar type to the frequency of encounters. The values of the number density $n_{* i}$ are derived for the $M_{V}$ intervals in brackets representing the stellar types, except for white dwarfs (WD) and giants.

\begin{tabular}{cccccc}
\hline Stellar type & $M_{V}{ }^{a}$ & $v_{* i}{ }^{b}$ & $v_{\odot i}{ }^{c}$ & $n_{* i}{ }^{d}$ & $f_{i}{ }^{e}$ \\
\hline B0 & $(-5.7,-0.2)$ & 14.7 & 18.6 & 0.06 & 0.005 \\
A0 & $(-0.2,1.3)$ & 19.7 & 17.1 & 0.27 & 0.03 \\
A5 & $(1.3,2.4)$ & 23.7 & 13.7 & 0.44 & 0.04 \\
F0 & $(2.4,3.6)$ & 29.1 & 17.1 & 1.42 & 0.15 \\
F5 & $(3.6,4.0)$ & 36.2 & 17.1 & 0.64 & 0.08 \\
G0 & $(4.0,4.7)$ & 37.4 & 26.4 & 1.52 & 0.22 \\
G5 & $(4.7,5.5)$ & 39.2 & 23.9 & 2.34 & 0.35 \\
K0 & $(5.5,6.4)$ & 34.1 & 19.8 & 2.68 & 0.34 \\
K5 & $(6.4,8.1)$ & 43.4 & 25.0 & 5.26 & 0.85 \\
M0 & $(8.1,9.9)$ & 42.7 & 17.3 & 8.72 & 1.29 \\
M5 & $(9.9,18.0)$ & 41.8 & 23.3 & 41.55 & 6.39 \\
WD & - & 63.4 & 38.3 & 3.00 & 0.72 \\
Giants & - & 41.0 & 21.0 & 0.43 & 0.06 \\
Totals & - & - & - & 68.33 & 10.525 \\
\hline
\end{tabular}

${ }^{a}$ Magnitude interval. ${ }^{b}$ Velocity dispersion in $\mathrm{km} \mathrm{s}^{-1} .{ }^{c}$ Sun's peculiar velocity in $\mathrm{km} \mathrm{s}^{-1}$. ${ }^{d}$ Number density in $10^{-3} \mathrm{pc}^{-3}$. ${ }^{e}$ Frequency of encounters within 1 pc of the Sun per Myr.

of Scalo (1986), based also on the difference between general and main sequence LF (see also Rana 1987 for the adopted Scalo's main sequence LF). Finally, for $M_{V}$ brighter than magnitude -1 we adopt a density of $8.0 \times$ $10^{-6} \mathrm{pc}^{-3}$ (Allen 1985). The contribution to the local density from other stellar types (e.g., supergiants, $M_{V}$ brighter than -6$)$ is not significant.

Table 8 gives the contribution of each stellar type to the frequency of encounters. Values for $v_{* i}$ and $v_{\odot i}$ are from Mihalas \& Binney (1981). From the results listed in this table we derive an expected frequency of encounters, $f_{\mathrm{e}}=10.5 \mathrm{Myr}^{-1}$ within $1 \mathrm{pc}$ of the Sun. Encounters with the more massive and slow moving early-type stars are much less frequent than with the more numerous later, low mass and high velocity, types. The stellar systems that potentially may cause the greatest dynamical perturbation on the Oort cloud represent only a small fraction of all star systems. In contrast, encounters with $M$ dwarfs represent about $73 \%$ of the total number of encounters. The contribution by white dwarfs is not very significant compared with that of $\mathrm{M}$ dwarfs. Even if we doubled the number density of white dwarfs, the total increase in the frequency of encounters would be less than $7 \%$.

\subsection{Frequency of encounters from the candidate stars}

In order to estimate the frequency of stellar encounters with the Sun, we also considered the predicted stellar passages obtained from the global potential model integrations using the Hipparcos data, and restricted by the following times $T_{\text {ca }}$ and distances $D_{\text {ca }}$ of closest approach:

$-1 \mathrm{Myr} \leq T_{\mathrm{ca}} \leq 1 \mathrm{Myr} ; \quad D_{\mathrm{ca}} \leq 5 \mathrm{pc}$.

We found a total of 92 star systems in our sample encountering the Sun, which matched the criteria in (33). The mean encounter velocity of these 92 systems with the Sun is $45.7 \mathrm{~km} \mathrm{~s}^{-1}$ (46.7 $\left.\mathrm{pc} \mathrm{Myr}^{-1}\right)$. If we take this velocity as a representative value, then the star systems satisfying the conditions (33) are expected to be currently located within a heliocentric distance of $\sim 50$ pc. In fact, we find that all 92 encounters are for star systems located within this distance.

There are 627 star systems with heliocentric distances up to $50 \mathrm{pc}$ in our sample of candidate stars, from which we derived the 92 passages satisfying (33). Figure 12 is a logarithmic plot of the cumulative number of predicted encounters within $5 \mathrm{pc}$ versus encounter distance, for a time interval of $\pm 1 \mathrm{Myr}$. The dashed line in the figure is a least-squares fit to the data, which has a slope of $2.2 \pm 0.2$, in agreement with the theoretically predicted slope of 2 . The encounters were derived from the systems with measured radial velocities, which constituted $64 \%$ of the 627 star systems. Assuming similar statistics for the total sample of 627 , we find a predicted value of $f_{\mathrm{s}}=2.3 \pm$ 0.2 star systems per Myr passing within 1 pc of the Sun.

\subsection{Frequency of encounters corrected for observational incompleteness}

The frequency of encounters derived from our candidate stars is a lower limit to the actual frequency because of the observational incompleteness in the Hipparcos data. The Hipparcos Catalogue is complete up to visual magnitude of about 7.3-9.0, depending on galactic latitude and spectral type, and has a limiting visual magnitude of $\sim 12$. Consequently, fainter stars are missed in our study.

To correct the frequency of encounters found above, we first estimate the number of star systems within $50 \mathrm{pc}$ of the Sun from the luminosity function constructed above, and then compare it with the number of star systems observed by the Hipparcos satellite. In the worst case, the Hipparcos catalogue is complete to a visual magnitude of $V_{\lim } \simeq 7.3$ for the entire sky, which is equivalent to a completeness to absolute magnitude $M_{V \lim } \simeq 4 \mathrm{mag}$ at 


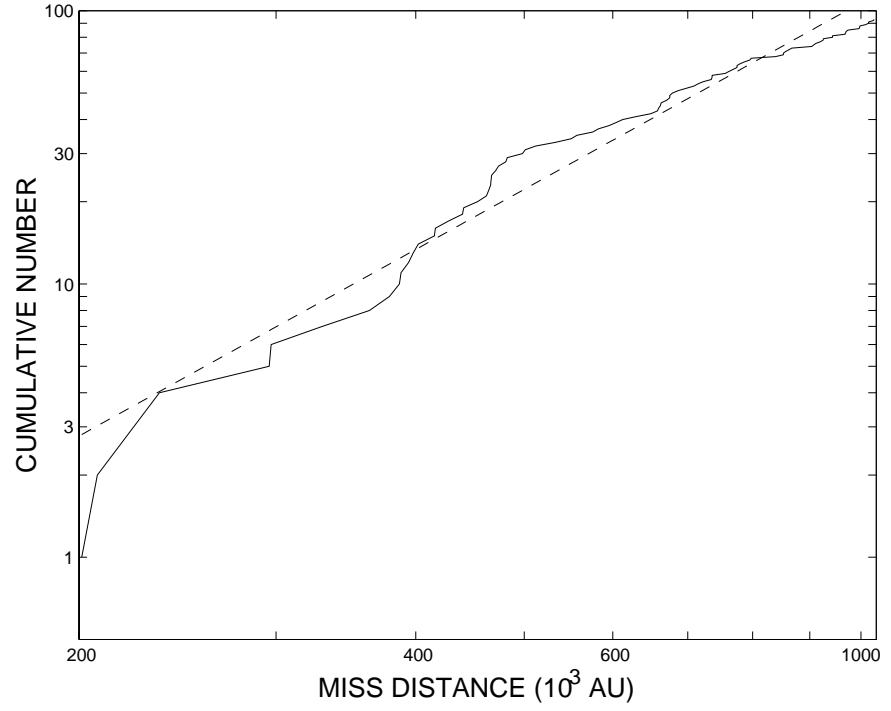

Fig. 12. Logarithmic plot of the cumulative number of predicted encounters versus miss distance $D_{\text {ca }}$ (in units of $10^{3} \mathrm{AU}$ ) The slope is $2.2 \pm 0.2$. The predicted encounter rate, corrected for our entire sample, is $2.3 \pm 0.2$ star systems per Myr passing within 1 pc of the Sun.

a radius of 50 pc. Thus, the Hipparcos Catalogue is expected to be incomplete in this case only if $M_{V}$ is fainter than $\sim 4$.

This incompleteness is shown in Fig. 13. The plot gives the number of star systems within $50 \mathrm{pc}$ estimated from the systemic LF, compared with the number detected by the Hipparcos satellite (black bar), as a function of $M_{V}$ (stellar type). Main sequence stars brighter than $M_{V}=$ -1 are not represented, since their number density $(8.0 \times$ $10^{-6} \mathrm{pc}^{-3}$ ) can be neglected.

The incompleteness of the Hipparcos data increases quickly as stars fainter than $M_{V} \sim 4$ are considered. Only $2 \%$ of the systems fainter than $M_{V}=9$ mag are detected. Encounters with a large number of low-mass $\mathrm{M}$ dwarfs are, thus, missed in our sample because of the intrinsic faintness of those stars. However, encounters with $\mathrm{M}$ dwarfs are not likely to perturb the Oort cloud significantly because of their low masses and typically high encounter velocities, unless there is a penetrating passage through the inner comet cloud.

The number of star systems within a heliocentric radius of 50 pc predicted by the systemic LF is $\sim 35800$, compared with $\sim 7000$ star systems observed by Hipparcos within this radius. Assuming statistical uncertainty in these numbers, this results in a factor $\sim 5.1 \pm 0.1$ incompleteness in the Hipparcos data. The frequency of encounters derived from our candidate stars is $2.3 \pm 0.2 \mathrm{Myr}^{-1}$. To correct this value for incompleteness, we multiply by the factor 5.1 estimated above. We obtain a corrected frequency of encounters $f_{\mathrm{c}}=11.7 \pm 1.3 \mathrm{Myr}^{-1}$ within $1 \mathrm{pc}$ of the Sun. This value is in agreement within the uncertainty with the frecuency of encounters $f_{\mathrm{e}}=10.5 \mathrm{Myr}^{-1}$ derived above from the LF.

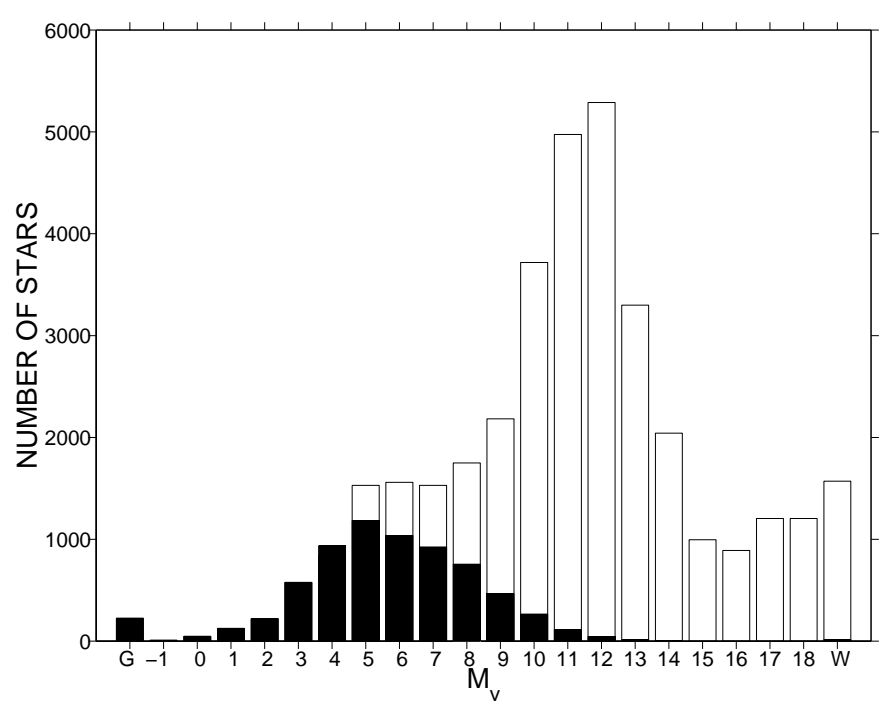

Fig. 13. Number of star systems within a heliocentric distance of $50 \mathrm{pc}$, in $1 \mathrm{mag}$ bins, derived from the systemic luminosity function. The black part of each bar is the number of star systems observed by Hipparcos. The bar to the left with the label $\mathrm{G}$ represents giant stars, whereas the bar to the right with the label $\mathrm{W}$ represents white dwarfs.

\section{Impact of future space-based astrometric missions on the search for close stellar passages}

Future space-based astrometric missions are expected to provide astrometric measurements with orders of magnitude better accuracy and observe a significantly greater number of stars than Hipparcos. Moreover, these missions will observe stars that are much fainter than the Hipparcos magnitude limit. The combination of both better accuracy and a much larger number of measured stars will make it possible to improve our knowledge of the frequency of encounters with the solar system and their dynamical effect on the Oort cometary cloud.

Several space-based astrometric missions are planned to be launched in the next few years: DIVA (Double Interferometer for Visual Astrometry, launch in 2004), FAME (Full-sky Astrometric Mapping Explorer, launch in 2004), SIM (Space Interferometry Mission, launch in 2009) and GAIA (Global Astrometric Interferometer for Astrophysics, launch no later than 2012). The comparison between these missions is summarized in Table 9 .

We consider two astrometric missions, GAIA and FAME, to study how future space-based missions may improve the search for close stellar passages. We choose these missions because they are expected to provide data for a larger number of stars (SIM, for instance, will observe a relatively small number of stars) and/or with better accuracy than the other astrometric missions.

\subsection{The GAIA mission}

GAIA, recently selected as ESA's Cornerstone 6 mission, will measure the positions and velocities of $\sim 10^{9}$ stars over a planned five-year mission. GAIA is the only 
Table 9. Comparison between Hipparcos, SIM, DIVA, FAME and GAIA.

\begin{tabular}{|c|c|c|c|c|c|}
\hline & Hipparcos & SIM & DIVA & FAME & GAIA \\
\hline Number of objects & $\sim 1.2 \times 10^{5}$ & $\sim 10^{4}$ & $\sim 3.5 \times 10^{7}$ & $\sim 4 \times 10^{7}$ & $\sim 10^{9}$ \\
\hline Magnitude limit & $V \approx 12$ & $V \approx 20$ & $V \approx 16$ & $V \approx 15$ & $V \approx 20$ \\
\hline Parallax accuracy & $\sim 1$ mas & $\sim 3 \mu \mathrm{as}$ & $\sim 250 \mu$ as $(V=10)$ & $\sim 50 \mu$ as $(V=9)$ & $\sim 4 \mu$ as $(V=12)$ \\
\hline & & & & $\sim 500 \mu$ as $(V=15)$ & $\sim 11 \mu$ as $(V=15)$ \\
\hline Proper motion acc. & $\sim 1{\operatorname{mas~} \mathrm{yr}^{-1}}^{-1}$ & - & $\sim 400 \mu$ as $\mathrm{yr}^{-1}(V=10)$ & $\sim 50 \mu$ as $\mathrm{yr}^{-1}(V=9)$ & $\sim 3 \mu$ as $\mathrm{yr}^{-1}(V=12)$ \\
\hline & & & & $\sim 500 \mu$ as $\mathrm{yr}^{-1}(V=15)$ & $\sim 8 \mu$ as $\mathrm{yr}^{-1}(V=15)$ \\
\hline Radial velocities & No & No & No & No & Yes \\
\hline
\end{tabular}

space-based mission that also proposes to provide radial velocity measurements for the target stars. The radial velocity accuracy will depend mainly on spectral type, rotational velocity, and metallicity, with an accuracy of $1-$ $10 \mathrm{~km} \mathrm{~s}^{-1}$ to a limiting magnitude $V \sim 17$.

To estimate how GAIA may improve the determination of stellar passages close to the Sun, we compare the uncertainty in the determination of the stellar encounters for our candidate stars due to the accuracy limits in the Hipparcos data with the uncertainty due to the expected accuracy limits of the GAIA data. We consider the 595 candidate Hipparcos stars with radial velocity measurements and we assume for these stars the errors in the parallaxes, proper motions and radial velocities expected from the measurements by GAIA. However, for those candidate stars for which we found radial velocity measurement errors in the literature smaller than the errors expected from GAIA, we adopt the errors found in the literature. All of the 595 candidate stars are brighter than visual magnitude 13. GAIA's expected accuracies for parallaxes and proper motions, 4 microarcsecond ( $\mu \mathrm{as}$ ) and $3 \mu \mathrm{as} \mathrm{yr}^{-1}$, respectively, for $V=12$, represent about two orders of magnitude improvement in accuracy over Hipparcos. If a given $\sigma_{i}$ represents the actual measurement uncertainty in parallax or proper motion in the Hipparcos data, we assume that the error for GAIA is $Q \sigma_{i}$, where $Q$ is the ratio between GAIA's and Hipparcos' theoretical uncertainties. Thus, the parallax uncertainty for GAIA is $0.004 \sigma_{i}$ and the proper motion uncertainty is $0.003 \sigma_{i}$. This implies that we assume, for instance, the same difficulties in the measurements by Hipparcos and by GAIA caused by the intrinsic physical properties of the target stars.

We define $\Delta D_{\mathrm{G}}$ as the uncertainty in the prediction of the miss distance due to the uncertainties in the parallaxes, proper motions and radial velocities (with the exceptions mentioned above for radial velocities) measured by GAIA. We also define $\Delta D_{\mathrm{H}}$ as the uncertainty in miss distance due to the uncertainties in the parallaxes and proper motions measured by Hipparcos as well as the uncertainties in the ground-based radial velocities from the literature. Both $\Delta D_{G}$ and $\Delta D_{\mathrm{H}}$ are calculated using Eq. (27) for the assumed uncertainties.

We plot the number of candidate stars as a function of the ratio $\Delta D_{\mathrm{H}} / \Delta D_{\mathrm{G}}$ in Fig. 14 . We find that all of the predicted passages are determined with better accuracy

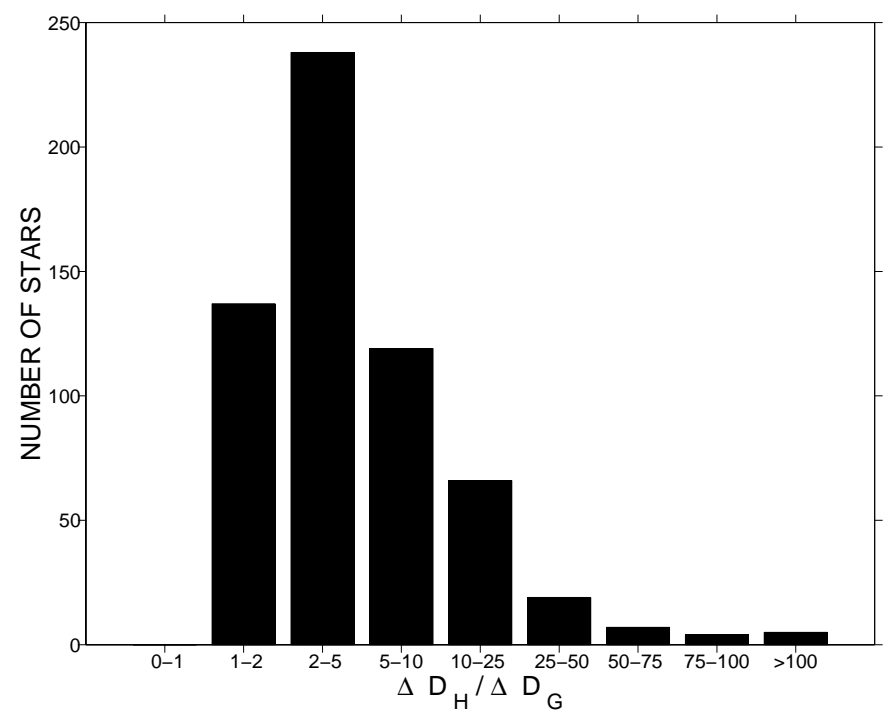

Fig. 14. Comparison between Hipparcos and GAIA in terms of the uncertainty in the determination of the closest approach distance to the Sun for the 595 candidate stars. The histogram gives the number of candidate stars as a function of the ratio between the uncertainty due to the measurement errors in the Hipparcos data and in the radial velocities, and the uncertainty due to the expected errors in the GAIA data.

assuming the uncertainty from GAIA. The error $\Delta D_{\mathrm{G}}$ is at least a factor of two smaller than the error $\Delta D_{\mathrm{H}}$ for $\sim 77 \%$ of the candidate stars. Miss distance errors are improved by more than one order of magnitude for $\sim 17 \%$ of the candidate stars (those stars with values of $\left.\Delta D_{\mathrm{H}} / \Delta D_{\mathrm{G}}>10\right)$.

We also estimate the same ratio $\Delta D_{\mathrm{H}} / \Delta D_{\mathrm{G}}$ for the 595 candidate stars, but taking into account only the uncertainties in the parallaxes and proper motions, and not the uncertainties in the radial velocity measurements. We plot the number of candidate stars as a function of this ratio in Fig. 15 with the same bins as in Fig. 14. With this assumption of perfect radial velocity measurements, the predicted passages using GAIA would be determined with much better accuracy. The predicted miss distances of practically all the candidate stars are determined with an error at least a factor of two smaller than the error due to the Hipparcos uncertainty. In this case, miss distance errors are improved by more than one order of magnitude 


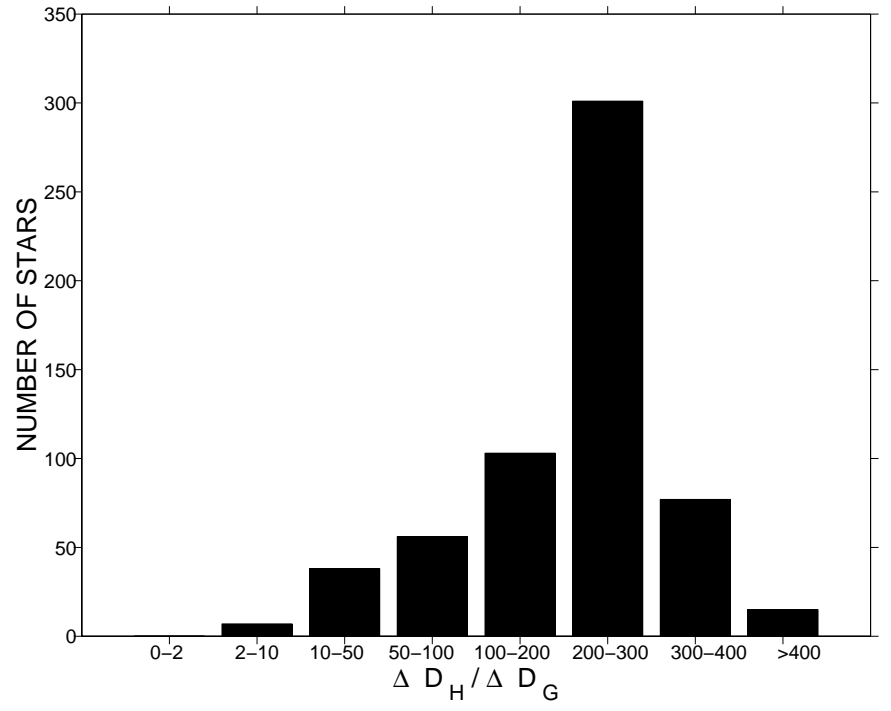

Fig. 15. Comparison between Hipparcos and GAIA in terms of the uncertainty in the determination of the closest approach distance to the Sun for the 595 candidate stars. The histogram gives the number of candidate stars as a function of the ratio between the uncertainty due to the measurement errors in the Hipparcos parallaxes and proper motions, and the uncertainty due to the expected errors in the GAIA parallaxes and proper motions. The difference with Fig. 14 is that the radial velocity measurement errors are not included here.

for $\sim 99 \%$ of the candidate stars, and by more than two orders of magnitude for $\sim 83 \%$ of the candidate stars.

From the comparison between Fig. 14, which includes the radial velocity uncertainties in the calculation, and Fig. 15, which does not include these errors, we see that the radial velocity uncertainties dominate the uncertainty in the predicted encounters over the parallax and proper motion errors. In either case, it is clear that GAIA will provide a significant improvement in the determination of close stellar encounters with the Sun, as compared with Hipparcos.

Finally, we estimate the errors in the miss distance considering the expected accuracies in parallax, proper motion and radial velocity by GAIA but, unlike above, not taking into account ground-based radial velocity measurements with better accuracy than those by GAIA. Comparing with Hipparcos results, we find that $85 \%$ of the predicted passages are determined with better accuracy assuming the uncertainty from GAIA. The errors are not improved by GAIA for the remaining $15 \%$ of the passages, though the difference is not large. The error in the determination of miss distances by GAIA is at least a factor of two smaller than the error due to the Hipparcos uncertainty for $\sim 65 \%$ of the candidate stars. Miss distance errors are improved by more than one order of magnitude for $\sim 12 \%$ of the candidate stars.

We plotted the distribution of ground-based radial velocity errors of the candidate stars in Fig. 2. About $40 \%$ of the stars in the plot have errors below $1 \mathrm{~km} \mathrm{~s}^{-1}$, the best radial velocity accuracy by GAIA. Furthermore, about $16 \%$ of the candidate stars have ground-based radial velocity measurements which are between three and ten times more accurate than GAIA's value of $1 \mathrm{~km} \mathrm{~s}^{-1}$. The very good accuracy of some of the ground-based radial velocities used in our study explains why GAIA, despite having better astrometric values, does not improve the uncertainty in some of the passages predicted when only the GAIA's accuracy in radial velocity is considered. It is clear that the combination of GAIA astrometric data with very accurate ground-based radial velocities provides the best results. However, such a combination will be limited to a relatively small percentage of future candidate stars, since GAIA is expected to observe a large number of stars for which no ground-based radial velocities will be available. Nevertheless, for close encounters predicted using GAIA's radial velocities, improved ground-based radial velocity data may eventually be obtained.

\subsection{Frequency of encounters and completeness}

We found that the observational incompleteness in the Hipparcos Catalogue is a significant source of uncertainty in the estimation of the frequency of encounters with the Sun. The future use of the GAIA data should overcome the problem of incompleteness for a large fraction of stellar types. Our estimate of the frequency of encounters was based on the star systems contained within a heliocentric distance of $50 \mathrm{pc}$. The distance modulus

$V-M_{V}=5 \log \left(\frac{d}{10}\right)$

where $d$ is the heliocentric distance of the star system (in pc), can be used to estimate the completeness of GAIA in terms of the absolute magnitude $M_{V}$. At a heliocentric distance $d=50 \mathrm{pc}$, the observational completeness of GAIA is given by $M_{V \lim }=V_{\lim }-3.495$, where $V_{\lim }$ is the magnitude limit of GAIA and $M_{V \lim }$ is the absolute limiting magnitude.

Using $V_{\text {lim }} \approx 20$ for GAIA and the equation above, GAIA will observe stars with completeness up to $M_{V \text { lim }} \approx$ 16.5 at 50 pc from the Sun. We can estimate the expected number of star systems observed by GAIA within a heliocentric distance of $50 \mathrm{pc}$, and compare it with the number of star systems observed by Hipparcos within this same distance. We consider the luminosity function adopted above and we assume that all stars or star systems brighter than $M_{V}=16.5$ can be detected by GAIA within 50 pc of the Sun. For fainter stars, we calculate the distance limit within which these stars can be detected by GAIA assuming the magnitude limit of $V=20$ for completeness. We then determine the number of stars or star systems located within this distance limit according to the luminosity function considered. The number of stars or star systems detected by GAIA is then compared with the prediction from the luminosity function.

We plot the number of stars or star systems within $50 \mathrm{pc}$ of the Sun as a function of $M_{V}$ (stellar type), in $1 \mathrm{mag}$ bins, given by the systemic luminosity function, in Fig. 16. The plot gives the number of stars detected by 


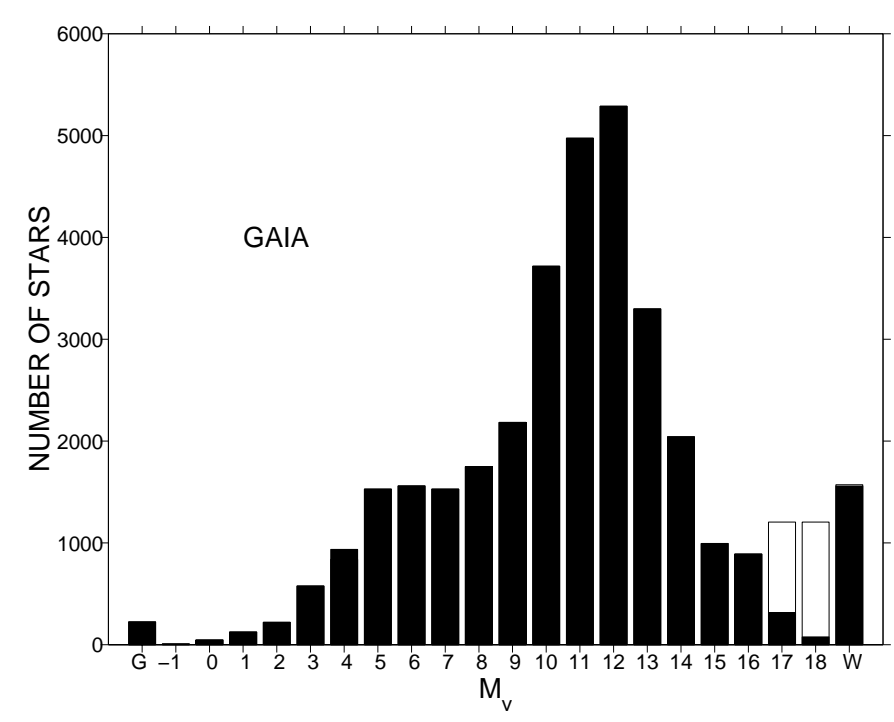

Fig. 16. GAIA completeness within 50 pc of the Sun. The plot is the same as in Fig. 13 (for the star systems observed by Hipparcos) but for GAIA.

GAIA (black part of each bar). This plot can be compared with the Hipparcos completeness shown in Fig. 13. We find that GAIA should observe at least $\sim 95 \%$ of the star systems within 50 pc, compared with only $\sim 20 \%$ of star systems within 50 pc observed by Hipparcos. This represents $\sim 33800$ star systems observed by GAIA within 50 pc, compared with $\sim 7000$ observed by Hipparcos within the same distance. GAIA represents a significant improvement in completeness compared with Hipparcos, which will result in a much better estimate of the frequency of stellar encounters with the Sun.

\subsection{The FAME mission}

FAME, a NASA MIDEX (medium-class Explorer) mission, is designed to perform an all-sky, astrometric survey that will create an accurate astrometric catalogue of $4 \times 10^{7}$ stars with visual magnitudes $5<V<15$. Unlike the GAIA mission, FAME will not measure the radial velocities of the target stars.

For FAME we proceed as in our study of GAIA, above, and consider the 595 candidate stars for which we have radial velocity measurements. For their astrometric values we assume different accuracies depending on the visual magnitude measured. For candidate stars with $V<9$, the errors in parallax or proper motion determined by FAME are expected to be better than $0.05 \sigma_{i}$, where $\sigma_{i}$ represents the measurement error in parallax or proper motion in the Hipparcos data. For candidate stars with $V>9$, the errors in parallax or proper motion determined by FAME increase, but to the limiting visual magnitude of the 595 candidate stars these errors are expected to be better than about $0.3 \sigma_{i}$.

Adopting these uncertainties for the candidate stars, we estimate the uncertainty $\Delta D_{\mathrm{F}}$ in the prediction of the miss distances due to the expected errors in the

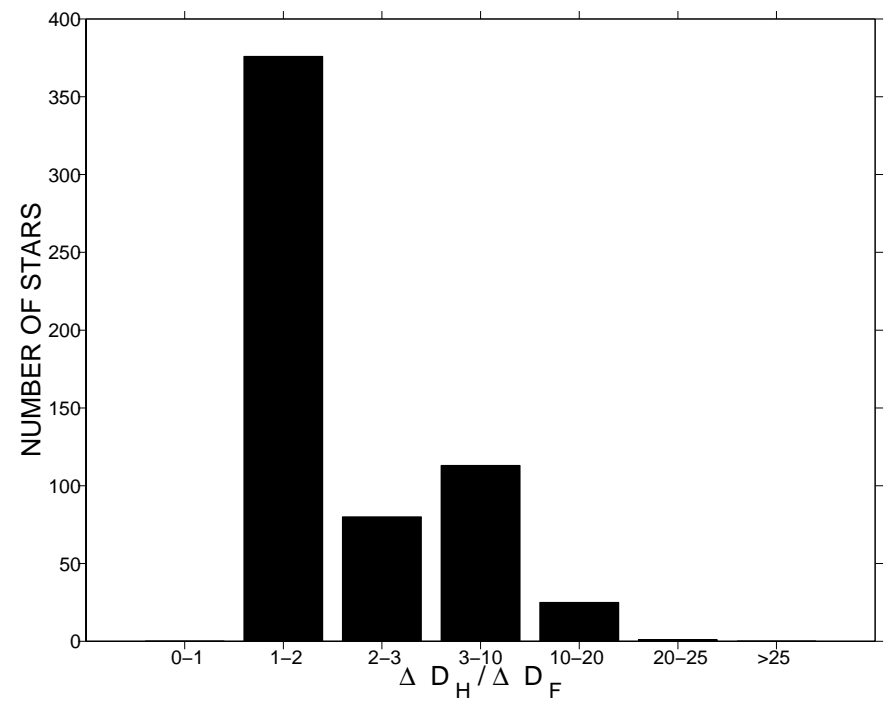

Fig. 17. Comparison between Hipparcos and FAME in terms of the uncertainty in the determination of the closest approach distance to the Sun for the 595 candidate Hipparcos stars. The histogram gives the number of candidate stars as a function of the ratio between the uncertainty due to the errors in the Hipparcos data and the uncertainty due to the expected errors in the FAME data. The errors in the ground-based radial velocity measurements are also included.

parallaxes and proper motions measured by FAME. The uncertainty from the Hipparcos errors is $\Delta D_{\mathrm{H}}$. Both uncertainties $\Delta D_{\mathrm{F}}$ and $\Delta D_{\mathrm{H}}$ are calculated using Eq. (27). In both cases the radial velocity measurement uncertainties are those we found in the literature.

We show the number of candidate stars as a function of the ratio $\Delta D_{\mathrm{H}} / \Delta D_{\mathrm{F}}$ in Fig. 17 . We find that the errors in the determination of miss distances by FAME are a factor of two or more smaller than the errors by Hipparcos for $\sim 37 \%$ of the candidate stars, compared with $\sim 77 \%$ of the candidate stars when GAIA is considered. Miss distance errors are improved by more than one order of magnitude using FAME data for $\sim 4 \%$ of the candidate stars, compared to $\sim 17 \%$ when GAIA is considered.

We also estimate $\Delta D_{\mathrm{F}}$ and $\Delta D_{\mathrm{H}}$ as above, but taking into account only the uncertainties for the parallaxes and proper motions and not the radial velocity measurement uncertainties. The number of candidate stars as a function of the ratio $\Delta D_{\mathrm{H}} / \Delta D_{\mathrm{F}}$ is shown in Fig. 18. In this case, the errors in the determination of miss distances by FAME are a factor of two or more smaller than the errors by Hipparcos for practically all the candidate stars. We also find that $\sim 72 \%$ of the candidate stars have one order of magnitude smaller errors with FAME, compared to $\sim 99 \%$ of the candidate stars with GAIA. From the comparison between Fig. 17 (with radial velocity errors) and Fig. 18 (with no radial velocity errors) we see that the radial velocity measurement uncertainties dominate the uncertainties in the predicted encounters.

FAME represents an improvement in the accuracy in the prediction of the miss distances compared to 


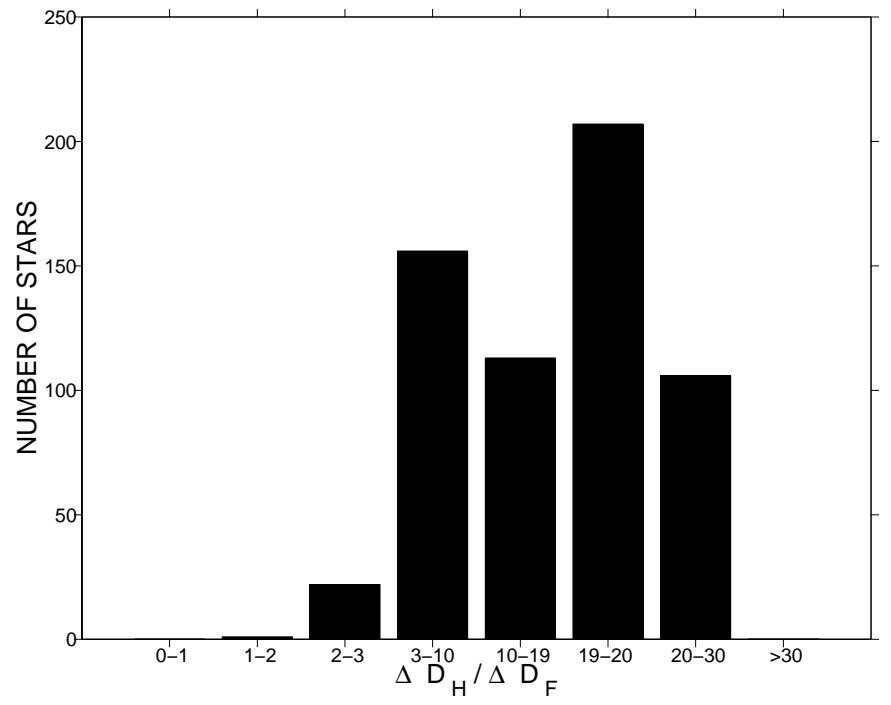

Fig. 18. Comparison between Hipparcos and FAME in terms of the uncertainty in the determination of the closest approach distance to the Sun for the 595 candidate Hipparcos stars. The histogram gives the number of candidate stars as a function of the ratio between the uncertainty due to the measurement errors in the Hipparcos data and the uncertainty due to the expected errors in the FAME data. The difference with respect to Fig. 17 is that the radial velocity measurement errors are not included here.

Hipparcos, though less than the improvement represented by GAIA. Nevertheless, FAME will play an important intermediate role since this mission should provide improved astrometric data compared to Hipparcos well in advance of GAIA. This would allow candidate stars for GAIA to be chosen, as well as radial velocity measurement programs to be initiated for candidate stars with no previous radial velocity measurements. This is especially important for long-period double and multiple systems, for which multiepoch measurements over several years may be required to determine their systemic radial velocities.

\subsection{Earth impacts and the search for close encounters}

A particularly interesting aspect of the search for stars that encountered the solar system in the past is the study of possible links between records of past impact events on Earth and comet showers. It has been suggested that the terrestrial record of impacts over the last $\sim 250 \mathrm{Myr}$ might be correlated with mass extinction events (see, e.g., Shoemaker \& Wolfe 1986).

Some terrestrial impact craters have been correlated with extinction events, though crater ages are often not known well enough. However, iridium anomalies, tektites, microtektites, impact glass and shocked minerals, which constitute markers of impact events in the stratigraphic column, can be used as independent evidence to support the timing of impact events.

At least two large impact structures can be correlated with the statigraphic records in the late Eocene about 36 Myr ago, the 100-km-diameter Popigai and the 90-km-diameter Chesapeake Bay impact craters. These two craters occurred synchronously within their age uncertainty. The age of the Popigai crater is $35.7 \pm 0.2 \mathrm{Myr}$ (Bottomley et al. 1997), and that of the Chesapeake Bay structure is $35.5 \pm 0.3 \mathrm{Myr}$ to $35.2 \pm 0.3 \mathrm{Myr}$ (Obradovich 1989; Poag \& Aubry 1995; Koeberl et al. 1996). For the last $150 \mathrm{Myr}$ these combined impact craters represent the third largest known impact energy flux peak and might be correlated with global biotic crises at the end of the Eocene (Montanari et al. 1985; Montanari et al. 1998). Farley et al. (1998) found geochemical evidence for a comet shower in the late Eocene; a significant increase in the flux of extraterrestial helium-3 to Earth. Helium-3 is a tracer of fine-grained interplanetary dust. Farley et al. suggested that the correlation between increased concentrations of helium-3 and large impacts indicates that the abundance of Earth-crossing objects and the dustiness of the inner solar system were simultaneously but only briefly enhanced. The flux of extraterrestial helium-3 began to increase before the impact events, reached a maxima coincident with those impacts, and then rapidly declined.

We estimated that the time horizon for our search for encounters with the solar system using Hipparcos data is roughly about $\pm 10 \mathrm{Myr}$ from the present time. The observational incompleteness in the Hipparcos Catalogue limits the determination of stellar encounters to within a few million years, beyond which the number of close passages missed by our search is very large. In contrast, GAIA may enhance this time interval to, at least, the past several tens of million years, as suggested by the comparison between Figs. 13 and 16. This is the time period required to cover the known cratering events in the late Eocene. Thus, the GAIA mission may provide sufficiently accurate data for a remarkably large number of stars to study the possible link between comet showers and records of past impact events on Earth.

We can estimate the number of main sequence stars with masses $\geq 1 M_{\odot}$ that can be detected, and their radial velocities measured, by GAIA. A close or penetrating passage of this massive a star through the Oort cloud could have caused a comet shower in the past. We adopt an absolute magnitude of $M_{V}=4.8$ (which corresponds to the Sun's value and which is equivalent to a lower mass limit) or brighter for this estimate. GAIA is complete to $V \approx 20$. However, to calculate the stellar trajectories we need radial velocity data, and the limiting magnitude for radial velocity measurements is $V \sim 17$, which we adopt for the calculation.

The effect of the interstellar absorption and scattering of light, $A_{V}$, cannot be neglected because of the large distances involved. We can consider $A_{V}$ to be proportional to the distance of the observed star, and assume a standard value of one magnitude per $\mathrm{kpc}$ for the dimming effect on the star's brightness. We consider the equation

$V-M_{V}=5 \log (d)-5+0.001 d$

where the distance $d$ is given in pc and $A_{V} \equiv 0.001 d$. Taking the values $V=17$ and $M_{V}=4.8$ above, and 


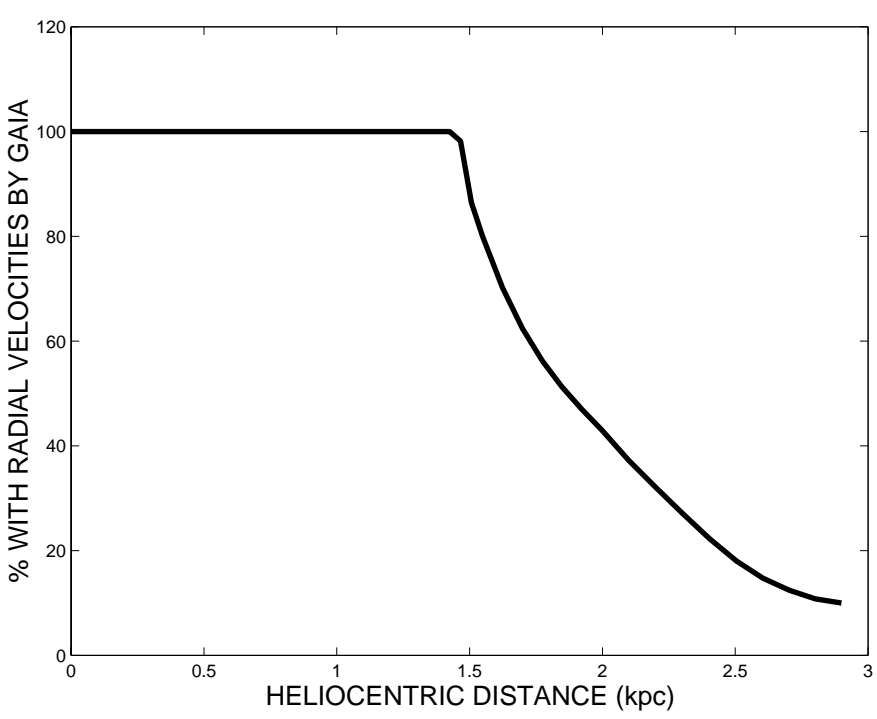

Fig. 19. Percentage of solar-type or more massive stars that will have their radial velocities measured by GAIA, as a function of the heliocentric distance in kpc. The expected completeness for GAIA stars with radial velocity measurements is to a distance of $\sim 1.4 \mathrm{kpc}$.

solving the equation, we find a distance of $d \simeq 1.4 \mathrm{kpc}$. Thus, GAIA should provide astrometric and radial velocity data for all solar-type or more massive stars located within a heliocentric distance of $d \simeq 1.4 \mathrm{kpc}$. The heliocentric distance to which GAIA's astrometry is observationally complete for solar-type or more massive stars, that is for the limiting magnitude of 20 , is $d \simeq 2.9 \mathrm{kpc}$. Between these two heliocentric distances only a percentage of these stars observed by GAIA will also have their radial velocities measured. We estimated this percentage based on the luminosity function adopted. The results are shown in Fig. 19. The number of stars with radial velocity measurements by GAIA begins to decrease for distances larger than $\sim 1.4 \mathrm{kpc}$, beyond which only the more massive, brighter stars will be measured.

The Hipparcos completeness for these solar-type or more massive candidate stars is to a distance of only $\sim 30-60$ pc, according to the Hipparcos magnitude limit of 7.3-9.0. GAIA, with $d \simeq 1.4 \mathrm{kpc}$, represents an improvement of a factor of $23-47$ with respect to Hipparcos, in terms of the limit distance within which completeness for the search for these candidates can be achieved. This corresponds to an increased search volume more than 12000 times that achieved by Hipparcos. In fact, this is a lower limit estimate since only a limited number of the Hipparcos star candidates have ground-based radial velocity measurements. The time horizon for completeness in the prediction of close passages in the past using GAIA is expected to be increased accordingly. It is difficult to estimate the typical time horizon for completeness because of the complexities of the stellar galactic orbits for the integration times involved. However, as a rough estimate, we can assume the same order of magnitude increase in the time horizon as in the increase in the distance horizon expected with the use of GAIA data. Therefore, the time horizon for the search for the candidate star that might have caused a comet shower can very likely be expanded by GAIA beyond the $\sim 36 \mathrm{Myr}$ ago date of the late Eocene impact events. Stars with slower encounter velocities are likely to have a bigger effect on the Oort cloud. Thus, perhaps the time horizon is even longer for these stars since they will not be as far from the Sun compared to faster moving stars. Of course, the transient nature of the stars must be taken into account, since only those with lifetimes beyond the $\sim 36 \mathrm{Myr}$ of the late Eocene events can be included in the search. Thus, massive early-type stars with short lifetimes should be ruled out. This type of stars, however, represents only a small fraction of the candidate stars.

An issue to be considered is that, with improved astrometric values, the ultimate limit on predicting close passages over the integration times required to account for the late Eocene event will be the galactic potential model, as well as the difficulty in recreating star-star and star-GMC encounters. Although it is difficult to quantify how accurately a stellar trajectory can be determined over such integration times, it seems clear that the uncertainty in the galactic parameters strongly limits the prediction capability. The largest source of error for encounters over timescales of several tens of Myr comes from the uncertainty in the density of matter in the galactic disk and the density variation, as shown above when we discussed the validity of our results. Thus, we need improved models of the Galaxy to reproduce better the actual gravitational potential if we want to predict accurately a close passage during the late Eocene event. However, better astrometric measurements can also lead to better potential models. This will improve our ability to integrate galactic orbits over longer timescales.

A more difficult problem is that of star-star and starGMC perturbations. In Paper I we showed that such perturbations can be neglected on timescales of a few Myr. However, they may become more significant over the 40 Myr timescale we would like to achieve with the FAME and GAIA data. Also, the short lifetimes of massive, early-type stars, which could act as significant perturbers, may make it impossible to recreate stellar trajectories with any reliability. It is even possible that a massive, early-type star was the late Eocene perturber, and that the star no longer exists, and thus can never be found.

\section{Conclusions}

1. We found that 156 candidate stars have passed or will pass within 5 pc of the Sun during about \pm 10 Myr from the present time, with most of them, $\sim 85 \%$, ocurring within a time interval of $\pm 3 \mathrm{Myr}$. The passages at large times are dominated by stars with the largest apparent brightness at closest approach, which suggests an observational bias that we interpreted as the result of observational incompleteness in the Hipparcos data. 
2. Within a time interval of about \pm 10 Myr from the present time, the predicted encounters are fairly well determined for most of the candidate stars. They are not altered significantly by the use of alternative galactic potential models or by varying the plausible values of the galactic parameters. It is the astrometric errors that ultimately limit the accuracy of the predicted encounters for most of our candidate stars over the time interval of integration considered. The time interval of about \pm 10 Myr from the present time is essentially an upper limit in our search for close stellar passages.

3. The most interesting result is the future passage of the star GL 710 through the outer Oort cloud. We determined a miss distance of $0.337 \pm 0.177 \mathrm{pc}$ in $1.36 \pm$ 0.04 Myr from the present for the passage of this star. These values are in excellent agreement with the predictions using the other galactic potential models, indicating that the prediction of this passage is not model dependent.

4. We estimated a value of 10.5 star systems per million years encountering the Sun within one pc based on a luminosity function constructed from data available in the literature. About $73 \%$ of the expected encounters are with late-type, low mass $M$ dwarfs. The frequency of encounters with the Sun was also estimated using the predicted passages for the Hipparcos candidate stars. We found a value of $2.3 \pm 0.2$ star systems per Myr passing within one pc of the Sun. This value represents a lower limit because of the observational incompleteness in the Hipparcos data. We corrected this value for incompleteness and derived a frequency of encounters with the Sun of $11.7 \pm 1.3$ star systems per Myr within a radius of one pc. Future measurements of radial velocities for the full sample of candidate stars are expected to improve the estimate of the lower limit above.

5 . Based on the predicted encounters for the 595 candidate Hipparcos stars, we found that GAIA and FAME would significantly decrease the uncertainty in the determination of the stellar encounters with the Sun. Radial velocity measurement errors would represent the dominant uncertainty factor in the passages predicted using data from these proposed missions. We expect a dramatic improvement in the determination of the frequency of encounters using GAIA data due to the much greater observational completeness estimated for that mission.

6. We suggest that GAIA may provide, for the first time, sufficiently accurate data and for a large enough number of stars to carry out a reliable study of the links between comet showers and past impact events on Earth. In particular, we considered the case in which records of multiple large impacts on Earth might be correlated with a comet shower during the late Eocene, $\sim 36 \mathrm{Myr}$ ago. GAIA should significantly increase the distance horizon that limits the search for past close encounters using Hipparcos data, and consequently expand the time horizon of our search beyond the $\sim 36 \mathrm{Myr}$ age of the late Eocene event. However, the galactic potential models may limit the prediction capability for encounters with large integration times.

Acknowledgements. We thank the suggestions made by an anonymous referee. This research was carried out in part at the Jet Propulsion Laboratory, California Institute of Technology, under contract with the National Aeronautics and Space Administration, and was supported in part by the Planetary Geology and Geophysics Program.

\section{References}

Allen, C. W. 1985, Astrophysical Quantities, 3rd. ed. (Atholone Press, London)

Amaral, L. H., \& Lépine, J. R. D. 1997, MNRAS, 286, 885

Antonov, V. A., \& Latyshev, I. N. 1972, in The Motion, Evolution of Orbits and Origin of Comets, ed. G. A. Chebotarev, E. I. Kazimirchak-Polonskaya, \& B. G. Marsden (Reidel, Dordrecht), 341

Asiain, R. 1998, Ph.D. Thesis, Universitat de Barcelona, Spain Avedisova, V. S. 1989, Ap, 30, 83

Bahcall, J. N. 1984a, ApJ, 276, 169

Bahcall, J. N. 1984b, ApJ, 287, 926

Bahcall, J. N., Flynn, C., \& Gould, A. 1992, ApJ, 389, 234

Bash, F. N. 1981, ApJ, 250, 551

Bertin, G., \& Lin, C. C. 1996, Spiral Structure in Galaxies: A Density Wave Theory (The MIT Press, Cambridge, MA)

Bienaymé, O., Robin, A. C., \& Crézé, M. 1987, A\&A, 180, 94

Binney, J., \& Tremaine, S. 1987, Galactic Dynamics (Princeton University Press, Princeton, NJ)

Blitz, L., Fish, M., \& Kulkarni, S. 1983, Science, 220, 1233

Bottomley, R., Grieve, R., York, D., \& Masaitis, V. 1997, Nature, 388, 365

Crézé, M., \& Mennessier, M. O. 1973, A\&A, 27, 281

Crézé, M., Chereul, E., Bienaymé, O., \& Pichon, C. 1998, A\&A, 329, 920

Dauphole, B., \& Colin, J. 1995, A\&A, 300, 117

Delfosse, X., Forveille, T., Mayor, M., et al. 1998, A\&A, 338, L67

Delhaye, J. 1965, in Galactic Structure, ed. A. Blaauw \& M. Schmidt (Univ. Chicago Press, Chicago), 61

Dehnen, W. 2000, AJ, 119, 800

Elmegreen, D. M. 1985, in The Milky Way Galaxy (Kluwer, Dordrecht) IAU Symp., 106, 255

The Hipparcos and Tycho Catalogues 1997, ESA SP-1200, Noordwijk

Farley, K. A., Montanari, A., Shoemaker, E. M., \& Shoemaker C. S. 1998 , Science, 280, 1250

Feast, M., \& Whitelock, P. 1997, MNRAS, 291, 683

Fischer, D. A., Marcy, G. W., Butler, R. P., Vogt, S. S., \& Apps, K. 1999, PASP, 111,50

Frogel, J. A., \& Gould, A. 1998, ApJL, 499, 219

García-Sánchez, J., Preston, R. A., Jones, D. L., et al. 1997, in Proceedings of the ESA Symp. Hipparcos - Venice 97, ed. B. Battrick (ESA SP-402, Noordwijk), 617

García-Sánchez, J., Preston, R. A., Jones, D. L., et al. 1999, AJ, 117, 1042

Georgelin, Y. M., \& Georgelin, Y. P. 1976, A\&A, 49, 57

Gerhard, O. E. 1999, in Galaxy Dynamics, ed. D. R. Merrit, M. Valluri, \& J. A. Sellwood (ASP, San Francisco), ASP Conf. Ser., 182, 307 
Gordon, M. A. 1978, ApJ, 222, 100

Gould, A., Bahcall, J. N., \& Flynn, C. 1997, ApJ, 482, 913

Hatzes, A. P., Cochran, W. D., McArthur, B., et al. 2000, ApJL, 544, L145

Henderson, A. P. 1977, A\&A, 58, 189

Hills, J. G. 1981, AJ, 86, 1730

Holmberg, J., Flynn, C., \& Lindegren, L. 1997, in Proc. of the ESA Symp., Hipparcos - Venice 97, ed. B. Battrick (ESA SP-402, Noordwijk), 721

Holmberg, J., \& Flynn, C. 2000, MNRAS, 313, 209

Humphreys, R. M., \& Larsen, J. A. 1995, AJ, 110, 2183

Hut, P., \& Tremaine, S. 1985, AJ, 90, 1548

Hut, P., Alvarez, W., Elder, W. P., et al. 1987, Nature, 329, 118

Jahreiss, H., \& Wielen, R. 1997, in Proc. of the ESA Symp., Hipparcos - Venice 97, ed. B. Battrick (ESA SP-402, Noordwijk), 675

Jeans, J. H. 1928, Astronomy and Cosmogony (Cambridge University Press, Cambridge)

Kerr, F. J., \& Lynden-Bell, D. 1986, MNRAS, 221, 1023

Koeberl, C., Poag, C. W., Reimold, W. U., \& Brandt, D. 1996, Science, 271, 1263

Kuijken, K., \& Gilmore, G. 1989, MNRAS, 239, 651

Kuzmin, G. G. 1956, AZh, 33, 27

Latham, D. W. 1985, in Stellar Radial Velocities, ed. A. G. D. Philip, \& D. W. Latham (Davis, Schenactady, N. Y.), IAU Colloq., 88, 21

Latham, D. W. 1992, in Complementary Approaches to Double and Multiple Star Research, ed. H. A. McAlister, \& W. I. Hartkopf (ASP, Chelsea), ASP Conf. Ser., 32, 158

Leggett, S. K., Ruiz, M. T., \& Bergeron, P. 1998, ApJ, 497, 294

Liebert, J., Dahn, C. C., \& Monet, D. G. 1988, ApJ, 332, 891

Lin, C. C., Yuan, C., \& Shu, F. H. 1969, ApJ, 155, 721

Lin, C. C., \& Bertin, G. 1985, in The Milky Way Galaxy (Kluwer, Dordrecht), IAU Symp., 106, 513

Luyten, W. J. 1979, The LHS Catalogue, 2d ed., Univ. Minnesota, Minneapolis

Mihalas, D., \& Routly, P. M. 1968, Galactic Astronomy (Freeman, San Francisco)
Mihalas, D., \& Binney, J. J. 1981, Galactic Astronomy, 2nd ed. (Freeman, San Francisco)

Mishurov, Y. N., \& Zenina, I. A. 1999, A\&A, 341, 81

Miyamoto, M., \& Nagai, R. 1975, PASJ, 27, 533

Montanari, A., Drake, R., Bice, D. M., et al. 1985, Geology, 13,596

Montanari, A., Bagatin, A. C., \& Farinella, P. 1998, Planetary and Space Science, 46, 271

Nelson, A. H., \& Matsuda, T. 1977, MNRAS, 179, 663

Obradovich, J. D., Snee, L. W., \& Izett, G. A. 1989, Geol. Soc. Am. Abstr. Programs, 21, 134

Olling, R. P., \& Merrifield, M. R. 1998, MNRAS, 297, 943

Pham, H-A. 1997, in Proc. of the ESA Symp. Hipparcos Venice 97, ed. B. Battrick (ESA SP-402, Noordwijk), 559

Plummer, H. C. 1911, MNRAS, 71, 460

Poag, C. W., \& Aubry, M-P. 1995, Palaios, 10, 16

Rana, N. C. 1987, A\&A, 184, 104

Reed, B. C. 1997, PASP, 109, 1145

Reid, M. J. 1993, ARA\&A, 31, 345

Reid, M. J., Readhead, A. C. S., Vermeulen, R. C., \& Treuhaft, R. N. 1999, ApJ, 524, 816

Rickman, H. 1976, Bull. Astron. Inst. Czechoslovakia, 27, 92

Ruiz, M. T., Takamiya, M. Y., Méndez, R., Maza, J., \& Wishnjewsky, M. 1993, AJ, 106, 2575

Scalo, J. 1986, Fundamentals of Cosmic Physics, vol. 11

Shoemaker, E. M., \& Wolfe, R. F. 1986, in The Galaxy and the Solar System, ed. R. Smoluchowski, J. N. Bahcall, \& M. S. Matthews (Univ. Arizona Press, Tucson), 338

Simonson, S. C. 1976, A\&A, 46, 261

Smoluchowski, R., \& Torbett, M. 1984, Nature, 311, 38

Stern, S. A., \& Shull, J. M. 1988, Nature, 332, 407

Trumpler, R. J., \& Weaver, H. F. 1953, Statistical Astronomy (Dover, New York)

Vallée, J. P. 1995, ApJ, 454, 119

Vyssotsky, A. N. 1946, PASP, 58, 166

Weissman, P. R. 1980, Nature, 288, 242

Weissman, P. R. 1996, Earth, Moon \& Planets, 72, 25

Yuan, C. 1969a, ApJ, 158, 871

Yuan, C. 1969b, ApJ, 158, 889 